\title{
Coupled diabatic potential energy surfaces for studying the nonadiabatic dynamics at conical intersections in angular resolved photodetachment simulations of $\mathrm{OHF}^{-} \rightarrow \mathrm{OHF}+e^{-}$
}

\author{
Susana Gómez-Carrasco \\ Unidad Asociada UAM-CSIC, Instituto de Matemáticas y Física Fundamental, CSIC, Serrano 123, \\ 28006 Madrid, Spain \\ Alfredo Aguado and Miguel Paniagua \\ Unidad Asociada UAM-CSIC, Departamento de Química Física, Facultad de Ciencias C-XIV, \\ Universidad Autónoma de Madrid, 28049 Madrid, Spain \\ Octavio Roncero ${ }^{\text {a) }}$ \\ Unidad Asociada UAM-CSIC, Instituto de Matemáticas y Física Fundamental, CSIC, Serrano 123, \\ 28006 Madrid, Spain
}

(Received 28 July 2006; accepted 22 September 2006; published online 26 October 2006)

\begin{abstract}
An energy-based method is proposed for the diabatization of the $\mathrm{OH}\left({ }^{2} \Pi\right)+\mathrm{F}\left({ }^{2} P\right) \rightarrow \mathrm{O}\left({ }^{3} P\right)$ $+\mathrm{HF}\left({ }^{1} \Sigma^{+}\right)$reaction. It is demonstrated that the diabatic representation obtained is regularized, i.e., the residual derivative couplings do not present singularities at the conical intersections appearing along the reaction path. This method only requires the knowledge of the $1,2^{3} A^{\prime \prime}$ and $1^{3} A^{\prime}$ eigenvalues and does not require any adjustable parameter. Thus, many convergence problems arising in other derivative-based diabatization methods are avoided, and the description of the configuration space along the reaction path is enormously simplified. Three-dimensional coupled diabatic energy surfaces are obtained by an interpolation procedure using $\approx 4000$ accurate ab initio points. The angular resolved photodetachment cross sections are obtained in the diabatic and adiabatic representations using a wave packet method. An excellent agreement is obtained with recent experimental data [D. M. Neumark, Phys. Chem. Chem. Phys. 7, 433 (2005)] for high electron kinetic energies where only the triplet electronic states contribute. (C) 2006 American Institute of Physics. [DOI: 10.1063/1.2363988]
\end{abstract}

\section{INTRODUCTION}

In atmospheric and interstellar chemistry open shell species, such as oxygen atoms and ions, play an important role because of their high reactivity. Collisions among those species involve several electronic states, very often degenerate either for reactants or products, which may cross along the minimum energy reaction path, leading to conical intersections.

In such situations, a detailed understanding of nonadiabatic reaction dynamics is quite complicated, due to the high number of electronic states involved and the possibility of multiple transitions among them. In collisional events, it is rather difficult to select a single initial electronic state, within a degenerate manifold, even with the use of external fields. One possible alternative is the photon excitation of the system to the states of interest. When the excitation forms an initial wave packet in the region of the transition state it is possible to obtain direct information about the region where the electronic rearrangement of a chemical reaction takes place: the so called transition state spectroscopy field of increasing interest. ${ }^{1-8}$ Since photon excitations obey well known selection rules, the analysis of angular distributions

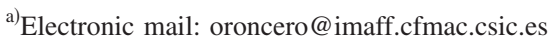

and rotational polarization of the fragments helps to determine the role of the electronic states involved. ${ }^{9}$

The reaction of hydrogen halides, $\mathrm{HX}$, with oxygen atoms contributes in the catalytic ozone destruction cycle $^{10}$ and, because its larger abundance, the chlorinated compounds have been the most widely studied, ${ }^{11-21}$ typically in single adiabatic potential energy surfaces (APESs). The realistic modeling of three-dimensional coupled diabatic potential energy surfaces (DPESs) for studying reaction dynamics requires high level $a b$ initio calculations of large complexity and the development of new diabatization methods. $\mathrm{O}+\mathrm{HF}$ may be considered as a benchmark model system for this kind of reactions because of its relatively simple electronic structure, albeit it presents most of the features of the $\mathrm{O}$ $+\mathrm{H} X$ family. Its first triplet states present several curve crossings along the minimum energy path at collinear configuration as shown in Fig. 1, leading to conical intersections of special interest in this work.

A source of experimental information about this system was obtained in the photoelectron detachment spectroscopic studies performed by Bradforth et al. ${ }^{22}$ In these experiments the $\mathrm{OHF}^{-}$anion, of linear equilibrium configuration, is excited by detaching an electron, and several electronic states of the neutral OHF system are reached in the region of the 


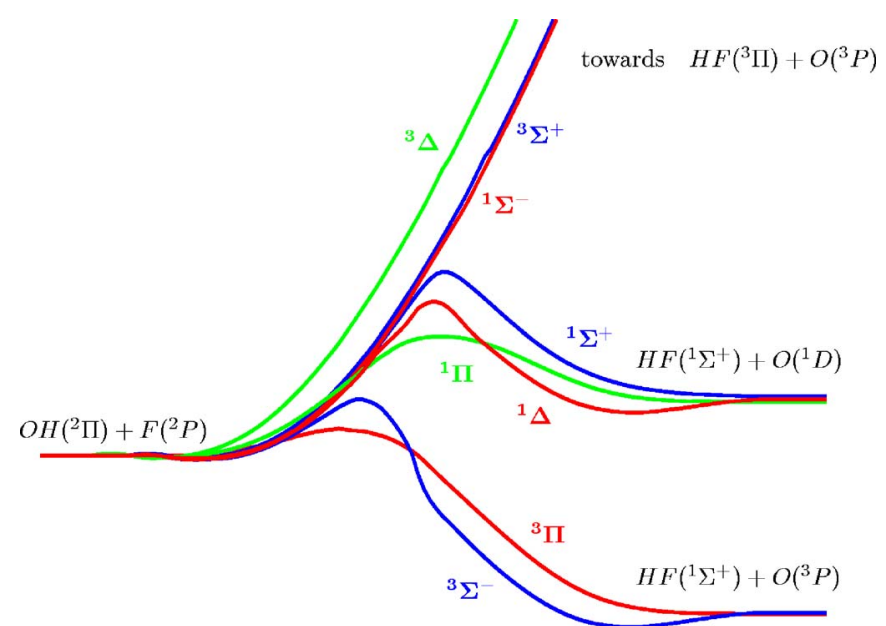

FIG. 1. Collinear minimum energy path for the electronic states correlating to the $\mathrm{OH}\left({ }^{2} \Pi\right)+\mathrm{F}\left({ }^{2} P\right)$ asymptote.

transition state. For this reason several theoretical simulations ${ }^{22,23}$ restricted to collinear OHF geometries and the ground electronic state were performed.

Later, three-dimensional PESs for the ground triplet $1{ }^{3} A^{\prime \prime}$ state ${ }^{24}$ and two first excited triplet states, $2{ }^{3} A^{\prime \prime}$ and $1^{3} A^{\prime}$, were obtained. ${ }^{25}$ The simulated photodetachment spectra, ${ }^{26}$ obtained using these three three-dimensional APESs of the lower triplet states, and four two-dimensional PESs for singlet states reproduced in an excellent way all the structures of the experimental photoelectron detachment spectrum. $^{22}$

However, the reaction cross section for the $\mathrm{OH}+\mathrm{F}$ collision on the excited triplet states was found to be too low. ${ }^{25}$ Thus, with only the ground triplet state contributing to the reaction the simulated rate constant is too small with respect to the experimental one. ${ }^{27}$ This could be attributed to inaccuracies of the APESs. However, the high quality of the $a b$ initio calculations and the accuracy of the fits indicate that this is probably not the only reason for the disagreement.

The reactivity must be deeply affected by the existence of nonadiabatic transitions due to conical intersections. At collinear geometries, the triplet states correlate with a ${ }^{3} \Sigma^{-}$ and two ${ }^{3} \Pi$ states. These states cross along the minimum energy path, as shown in Fig. 1. The crossing seams between the ${ }^{3} \Sigma^{-}$and ${ }^{3} \Pi$ states are clearly shown in Fig. 2, at collinear geometry. As long as the system bends, the ${ }^{3} \Sigma^{-}$state interacts with ${ }^{3} \Pi$ states, yielding two ${ }^{3} A^{\prime \prime}$ states and one ${ }^{3} A^{\prime}$ state, and $\Sigma$ - $\Pi$ vibronic effects should appear, which could be responsible for increasing reactivity in the excited triplet states.

The exploration of this nonadiabatic mechanism has become accessible by two new sets of photodetachment spectroscopy experiments, which allow the probing of conical intersections as has already been reported. ${ }^{28-30}$ In one side, Neumark published ${ }^{31}$ the photoelectron detachment spectra recorded some years before ${ }^{32}$ at two geometries with respect to the polarization vector of the incident light. Since the equilibrium geometry of the parent $\mathrm{OHF}^{-}$anion is collinear, such data correspond to parallel and perpendicular transitions, thus allowing the detection of two sets of electronic states separately, as will be treated in detail in the present

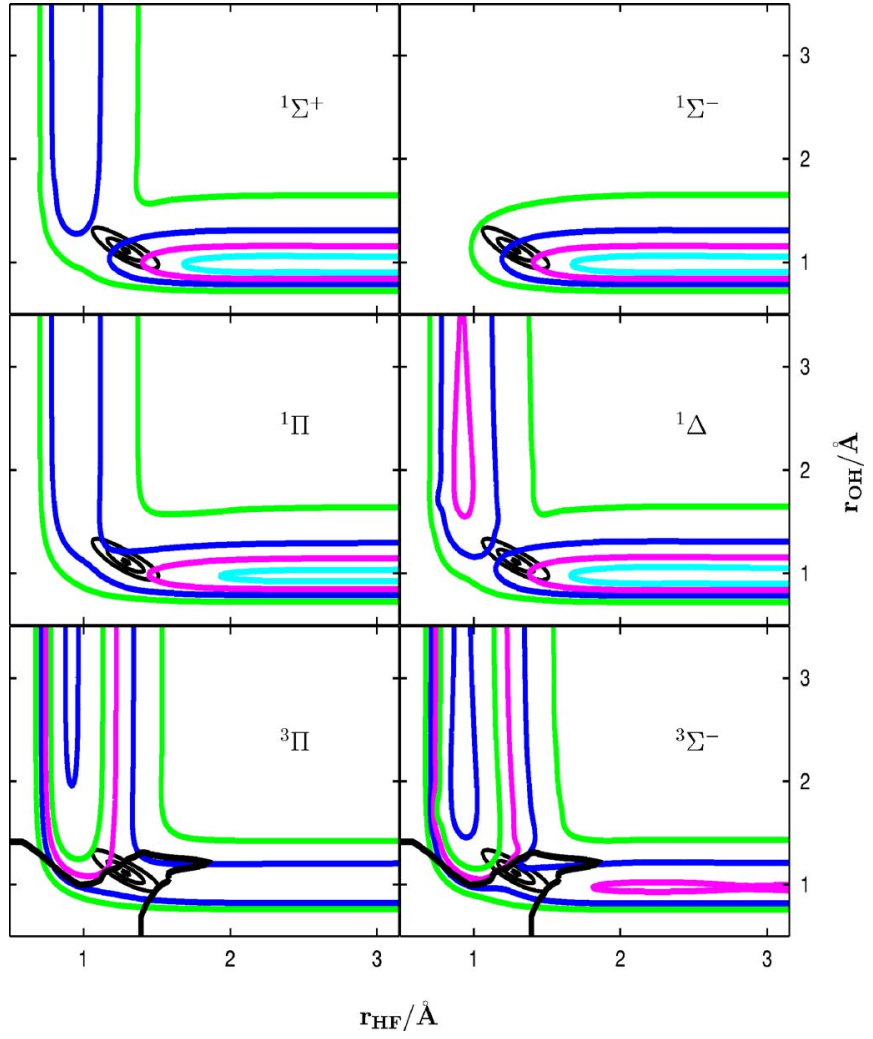

FIG. 2. Contour plots of the first electronic states of the OHF system at collinear geometry. The contours are $-1.4,-0.6,0,0.8$, and $2 \mathrm{eV}$ for triplets and $0.1,0.5,1.3$, and $3 \mathrm{eV}$ for singlets. All energies referred to the $\mathrm{OH}\left({ }^{2} \Pi\right)+\mathrm{F}\left({ }^{2} P\right)$ asymptote. The initial wave packet, essentially corresponding to the ground vibrational state of $\mathrm{OHF}^{-}$anion, is shown in all the panels in black. Also, for the lower triplet states, the crossing seam is shown in black lines.

work. Moreover, since the initial wave packet is sitting on the top of the two conical intersections, see Fig. 2, these spectra provide direct spectroscopic data about the region of the crossings. Second, Deyerl and Continetti ${ }^{33}$ and Continetti $^{34}$ have detected in coincidence the electron and neutral fragments. Since the formation of products requires that the wave packet passes through the conical intersections, such kind of experiments would provide information about nonadiabatic transitions induced by conical intersections in the reaction dynamics.

In this work, coupled DPESs are developed to describe the three lowest triplet states of the $\mathrm{OH}+\mathrm{F} \rightarrow \mathrm{O}+\mathrm{HF}$ reaction in Sec. II. The photodetachment spectra are simulated and compared with the experimental data recently measured, ${ }^{31,32}$ as described in Sec. III. Special emphasis is done regarding the nonadiabatic effects introduced by the conical intersections present in this system. Finally, Sec. IV is devoted to some conclusions.

\section{COUPLED DIABATIC POTENTIAL ENERGY SURFACES}

The Born-Oppenheimer approximation fails at conical intersections because nonadiabatic derivative couplings diverge where two states cross, according to ${ }^{35}$ 


$$
\left\langle\varphi_{i}\left|\nabla_{\mathbf{Q}}\right| \varphi_{i^{\prime}}\right\rangle=\frac{\left\langle\varphi_{i}\left|\nabla_{\mathbf{Q}} H_{e}\right| \varphi_{i^{\prime}}\right\rangle}{E_{i}(\mathbf{Q})-E_{i^{\prime}}(\mathbf{Q})}
$$

where $E_{i}$ and $E_{i^{\prime}}$ are the electronic eigenvalues of the electronic Hamiltonian $H_{e}$ and the vector $\mathbf{Q}$ specifies the nuclear configuration. In such situation it is convenient to transform from the adiabatic, $\left\{\varphi_{i}\right\}$, to a diabatic representation, $\left\{\phi_{i}\right\}$, through an unitary transformation as ${ }^{36-38}$

$$
\phi_{j}=\sum_{i} \varphi_{i} T_{i j}(\mathbf{Q}) .
$$

In the diabatic representation the derivative couplings become

$$
\left\langle\phi_{j}\left|\nabla_{\mathbf{Q}}\right| \phi_{j^{\prime}}\right\rangle=\sum_{i^{\prime}} T_{j i^{\prime}}\left\{\left(\nabla_{\mathbf{Q}} T_{i^{\prime} j^{\prime}}\right)+\sum_{i} T_{i j^{\prime}}\left\langle\varphi_{i^{\prime}}\left|\nabla_{\mathbf{Q}}\right| \varphi_{i}\right\rangle\right\} .
$$

As noted by Smith $^{36}$ and Baer, ${ }^{37,38}$ the derivative couplings in the diabatic basis vanish if the transformation matrix $T$ is such that the differential equation appearing between braces in the right-hand side of Eq. (2) is equal to zero. Such condition is only strictly fulfilled when the vector $\mathbf{Q}$ restricts to a single coordinate, as it is the case of diatomic system. In this case conical intersections do not appear, but it is still advantageous to work in the diabatic representation because it avoids the appearance of narrow peaks in the derivative nonadiabatic couplings.

For polyatomic systems, however, there is no strict diabatic representation because, in general, curl condition is not fulfilled ${ }^{37,39}$ and it is not possible to eliminate all derivative couplings in all the configuration space. Nevertheless, it is convenient to transform to a quasidiabatic representation where the derivative couplings are somehow minimized. These quasidiabatic representations are not unique since they depend on the path chosen to solve the first order differential equation to eliminate one/some first derivative matrices. ${ }^{36,37}$ To avoid this arbitrariness, it is then required to add to the diabatic matrix the residual derivative couplings, which are expected to be much smaller than in the adiabatic representation. When there are conical intersections it is then crucial to eliminate the singularity of the derivative couplings at crossings. This can be accomplished by a "regularization" procedure, as shown by Thiel and Köppel, ${ }^{40}$ producing regular diabatic states whose derivative coupling matrices do not show singularities at crossing, but instead vary smoothly.

The methods for quasidiabatization can be classified in three groups, as recently reviewed: ${ }^{35}$ derivative-based methods, ${ }^{36-38,41}$ property-based methods, ${ }^{42}$ and energy-based methods. These methods involve a decreasing computational effort. Derivative-based methods involve the calculation of nonadiabatic derivative matrix elements, which requires highly accurate electronic wave functions. Property-based methods also require the calculation of wave functions to evaluate some property, such as electric dipole, which is assumed to change smoothly with the nuclear configuration and is diagonal in the diabatic representation. Finally, energy-based methods only require the knowledge of the ei- genvalues and are based on a good knowledge of the system and very frequently are assumed to require some adjustable parameters.

The construction of coupled DPESs of ab initio accuracy for reaction dynamics is a difficult task because it requires the calculation of several electronic states, and their mutual nonadiabatic couplings, using high correlation methods over all the configuration space, from reactants to products. In this section we shall propose a simple energy-based diabatization method based on a simplified model. The diabatic Hamiltonian matrix elements are directly obtained from adiabatic eigenvalues and do not depend on any adjustable parameter. This procedure is compared with a derivative-based method $^{43}$ as it is implemented in the MOLPRO package. ${ }^{44}$ Furthermore, we shall study the behavior of the derivative matrix elements in the diabatic representation near the conical intersection to check if it is a "regularized" diabatic representation. Finally, we shall describe a global threedimensional DPES and check its accuracy by analyzing the differences of its adiabatic eigenvalues with those obtained in highly accurate internally contracted multireference configuration interaction (ic-MRCI) calculations. ${ }^{45,46}$

\section{A. Electronic model}

The reaction to be studied is

$$
\mathrm{OH}\left({ }^{2} \Pi\right)+\mathrm{F}\left({ }^{2} P\right) \rightarrow \mathrm{HF}\left({ }^{1} \Sigma^{+}\right)+\mathrm{O}\left({ }^{3} P\right),
$$

while the third rearrangement channel, yielding OF products, is energetically closed. The transfer of the $\mathrm{H}$ atom is produced at short distances between the heavier atoms. HF and $\mathrm{OH}$ diatomic fragments have a significant ionic character and, at a zero order approach, they can be considered as $\mathrm{H}^{+} \mathrm{F}^{-}$and $\mathrm{O}^{-} \mathrm{H}^{+}$. Under this assumption, the reaction can be described as

$$
\mathrm{O}^{-}\left({ }^{2} P\right) \mathrm{H}^{+}+\mathrm{F}\left({ }^{2} P\right) \rightarrow \mathrm{H}^{+} \mathrm{F}^{-}\left({ }^{1} S\right)+\mathrm{O}\left({ }^{3} P\right) .
$$

The reaction can then be viewed as a proton transfer, from $\mathrm{O}^{-}$to $\mathrm{F}$ atom, while an electron-hole is transferred back from $\mathrm{F}$ to $\mathrm{O}^{-}$(equivalent to an electron transfer from $\mathrm{O}^{-}$to F). The triatomic system can then be treated as a proton orbiting the $\mathrm{OF}^{-}$fragment. Since neutral $\mathrm{F}$ and $\mathrm{O}$ atoms can be treated as one- and two-electron-hole systems, respectively, consequently $\mathrm{OF}^{-}$can be treated as a two-electronhole diatomic fragment. In addition, the $\mathrm{OF}$ vector, $\mathbf{R}_{\mathrm{OF}}$, thus constitutes a quite natural quantization axis, and the much lighter proton and electron-holes move around it.

The electronic Hamiltonian of the triatomic system can then be factorized as $H_{e}=H_{0}+V_{e} . H_{0}$ describes the $\mathrm{OF}^{-}$diatomic fragment of cylindrical symmetry. The coupling term $V_{e}$ describes the interaction of the proton with the nucleus of $\mathrm{OF}^{-}$, also of cylindrical symmetry, plus the electrostatic interaction among the electron-holes and the proton. Each of these two terms can be expanded as ${ }^{47}$

$$
\frac{1}{\left|\mathbf{R}_{\mathrm{H}}-\boldsymbol{\rho}_{i}\right|}=\sum_{\ell=0}^{\infty} a_{\ell}\left(R_{\mathrm{H}}, \rho_{i}\right) \sum_{m=-\ell}^{\ell} Y_{\ell m}^{*}\left(\hat{R}_{\mathrm{H}}\right) Y_{\ell m}\left(\hat{\rho}_{i}\right),
$$

where $\mathbf{R}_{\mathrm{H}}$ (with $\hat{R}_{\mathrm{H}} \equiv(\gamma, \chi)$ ) and $\boldsymbol{\rho}_{i}$ are the position vectors of the proton and each of the two-electron-holes with respect 
to the OF center of mass, respectively. Each $1 /\left|\mathbf{R}_{\mathrm{H}}-\boldsymbol{\rho}_{i}\right|$ term produces a change in the projection of the electronic angular momentum $\lambda_{i}$ associated with $\boldsymbol{\rho}_{i}$. However, no single term changes simultaneously the projection of the two holes, $\lambda_{1}$ and $\lambda_{2}$.

The electronic spin part can be separated, and the basis set functions are classified by the projection of the orbital angular momentum on the OF axis, $\Lambda$. These functions are built as linear combination of atomic electronic functions, $\ell_{\lambda}^{A}$, where $\ell$ is the electronic orbital momentum and the superscript $A$ refers to the atomic origin. A crude model can be constructed by considering a small electronic basis, formed by $\Lambda=0$ functions,

$$
\begin{aligned}
& p_{1}^{\mathrm{O}}(1) p_{-1}^{\mathrm{F}}(2)-p_{1}^{\mathrm{O}}(2) p_{-1}^{\mathrm{F}}(1) \\
& -p_{-1}^{\mathrm{O}}(1) p_{1}^{\mathrm{F}}(2)+p_{-1}^{\mathrm{O}}(2) p_{1}^{\mathrm{F}}(1) \text { for reactants, }
\end{aligned}
$$

$p_{1}^{\mathrm{O}}(1) p_{-1}^{\mathrm{O}}(2)-p_{1}^{\mathrm{O}}(2) p_{-1}^{\mathrm{O}}(1) \quad$ for products

(under reflection through the $x-z$ body-fixed frame these functions have an eigenvalue of -1 , giving rise to ${ }^{3} \Sigma^{-}$ states), $\Lambda=1$ functions,

$$
\begin{aligned}
& p_{1}^{\mathrm{O}}(1) p_{0}^{\mathrm{F}}(2)-p_{1}^{\mathrm{O}}(2) p_{0}^{\mathrm{F}}(1) \text { for reactants, } \\
& p_{1}^{\mathrm{O}}(1) p_{0}^{\mathrm{O}}(2)-p_{1}^{\mathrm{O}}(2) p_{0}^{\mathrm{O}}(1) \text { for products, }
\end{aligned}
$$

and $\Lambda=-1$ functions, which are equivalent to those written for $\Lambda=1$, but with $p_{-1}^{\mathrm{O}}$ instead of $p_{1}^{\mathrm{O}}$.

Within each $\Lambda$ subspace there are two functions, one describing each rearrangement channel. This situation can be more simplified by considering the lowest eigenfunction in each $\Lambda$ subspace, thus reducing the problem to three states, $\left(\phi_{-1}, \phi_{0}, \phi_{1}\right)$, considered to form the diabatic representation, one per $\Lambda$ value.

In this $3 \times 3$ subspace, the diabatic states are coupled through the $V_{e}$ potential term. The resulting Hamiltonian matrix is completely analogous to those used before for treating $\Sigma$-П vibronic effects ${ }^{48}$ and becomes

$$
\begin{array}{r}
\mathbf{H}_{e} \\
=\left(\begin{array}{ccc}
E_{-1}(r, R, \gamma) & V_{1}(r, R, \gamma) e^{i \chi} & 0 \\
V_{1}(r, R, \gamma) e^{-i \chi} & E_{0}(r, R, \gamma) & -V_{1}(r, R, \gamma) e^{i \chi} \\
0 & -V_{1}(r, R, \gamma) e^{-i \chi} & E_{1}(r, R, \gamma)
\end{array}\right),
\end{array}
$$

where $E_{1}=E_{-1}$. The direct coupling between $\phi_{1}$ and $\phi_{-1}$ is zero because each proton-electron-hole interaction term, $1 /\left|\mathbf{R}_{\mathrm{OF}}-\boldsymbol{\rho}_{i}\right|$, cannot change the projection of the orbital angular momentum of the two electrons at the same time. Indirect couplings could appear due to some other states, but in the present simple model, with three diabatic states, this is not possible. Moreover, using Eq. (5) the matrix elements of the $\hat{V}_{e}$ coupling term are $V_{1}(r, R, \gamma) \propto \sin \gamma$, as $\gamma \rightarrow 0$ or $\pi$.

Since the system presents a plane of symmetry, this diabatic representation can be transformed to a new symmetry adapted diabatic basis $\left(\phi_{Y}, \phi_{Z}, \phi_{X}\right)$, in which the transformation brings $\mathbf{R}_{\mathrm{H}}$ to the $x-z$ plane as ${ }^{48}$

$$
\left(\phi_{Y} \phi_{Z} \phi_{X}\right)=\left(\begin{array}{lll}
\phi_{-1} & \phi_{0} & \phi_{1}
\end{array}\right)\left(\begin{array}{ccc}
e^{i \chi} / \sqrt{2} & 0 & e^{i \chi} / \sqrt{2} \\
0 & 1 & 0 \\
-e^{-i \chi} / \sqrt{2} & 0 & e^{-i \chi} / \sqrt{2}
\end{array}\right)
$$

In this representation the Hamiltonian becomes block diagonal,

$$
\widetilde{\mathbf{H}}_{e}=\left(\begin{array}{ccc}
E_{1}(r, R, \gamma) & V_{1}(r, R, \gamma) \sqrt{2} & 0 \\
V_{1}(r, R, \gamma) \sqrt{2} & E_{0}(r, R, \gamma) & 0 \\
0 & 0 & E_{1}(r, R, \gamma)
\end{array}\right),
$$

whose eigenvalues

$$
E_{ \pm}=\left(E_{1}+E_{0} \pm \sqrt{\left(E_{1}-E_{0}\right)^{2}+8 V_{1}^{2}}\right) / 2 \text { and } E_{1}
$$

are obtained in the $a b$ initio calculations.

\section{B. Diabatization method}

The block diagonal Hamiltonian of Eq. (8) only depends on three parameters, $E_{0}, E_{1}$, and $V_{1}$. In the present case, $E_{1}$ corresponds to the eigenvalues of the $1^{3} A^{\prime}$ electronic state. $E_{0}$ and $V_{1}$ are obtained from $E_{-}$and $E_{+}$, the eigenvalues of $1^{3} A^{\prime \prime}$ and $2{ }^{3} A^{\prime \prime}$ states, respectively, as

$$
\begin{aligned}
& E_{0}=E_{+}+E_{-}-E_{1}, \\
& V_{1}^{2}=\left[\left(E_{+}-E_{-}\right)^{2}-\left(E_{1}-E_{0}\right)^{2}\right] / 8 .
\end{aligned}
$$

Thus the three parameters determining the diabatic Hamiltonian can be directly obtained from $a b$ initio calculations, at the desired level of accuracy, and do not depend on any adjustable parameter. Unless otherwise stated, all the $a b$ initio calculations have been performed using the ic-MRCI method with the MOLPRO suite of programs, ${ }^{44}$ as will be described below.

The diabatic-to-adiabatic transformation matrix can be expressed in terms of the mixing angle $\alpha$ for the two ${ }^{3} A^{\prime \prime}$ states. In general, the residual first derivative couplings in the diabatic representation can be expressed in terms of the nonadiabatic coupling matrix elements, between the adiabatic functions $\varphi_{-}$and $\varphi_{+}$, as

$$
\left\langle\phi_{0}\left|\frac{\partial}{\partial X}\right| \phi_{1}\right\rangle=\left\langle\varphi_{-}\left|\frac{\partial}{\partial X}\right| \varphi_{+}\right\rangle-\frac{\partial \alpha}{\partial X},
$$

where $X \equiv \gamma, R_{\mathrm{OH}}, R_{\mathrm{HF}}$ refers to the internal coordinates used. In the present energy-based method the mixing angle, $\alpha^{E}$, is directly calculated as

$$
\tan 2 \alpha^{E}=\frac{2 V_{1}}{E_{0}-E_{1}},
$$

where $E_{0}, E_{1}$, and $V_{1}$ are obtained from the adiabatic eigenvalues using Eq. (10). It is interesting to analyze the residual derivative couplings resulting from such election of the mixing angle.

For this purpose, we shall compare with the derivativebased method of Ref. 43, implemented in the MOLPRO suite of programs, in which the $\alpha^{D}$ mixing angle is obtained from the CI overlap matrix, which is equivalent to solving 


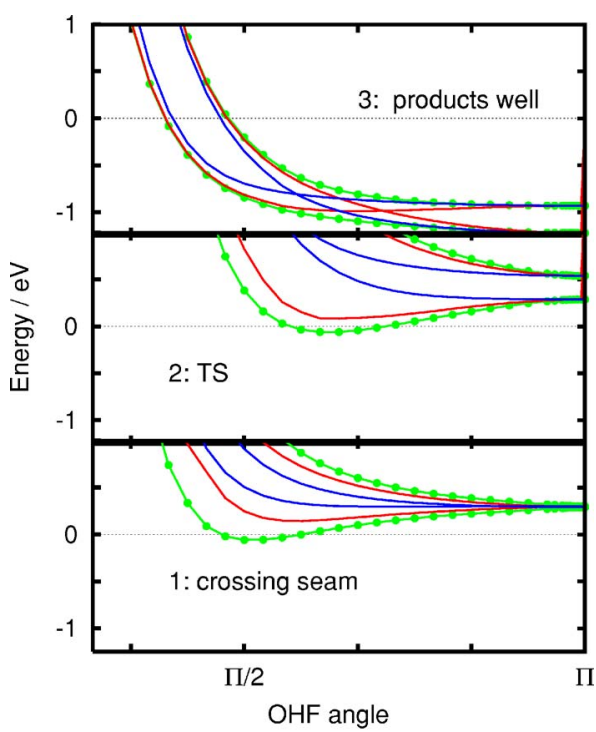

FIG. 3. Diabatic energies obtained using Eq. (10) (in red) and the derivative-based method of Ref. 43 (in blue) at three different points along the reaction path: (bottom panel) at $R_{\mathrm{OH}}=2$ a.u. and $R_{\mathrm{HF}}=3$ a.u. close to the crossing seam, (middle panel) at $r_{\mathrm{OH}}=2$ a.u. and $R_{\mathrm{HF}}=2.4$ a.u. near the saddle point, and (top panel) at $r_{\mathrm{OH}}=3$ a.u. and $R_{\mathrm{HF}}=1.8$ a.u. in the product channel. The adiabatic eigenvalues are also included for comparison.

$$
\frac{\partial \alpha^{D}}{\partial \gamma}=\left\langle\varphi_{-}\left|\frac{\partial}{\partial \gamma}\right| \varphi_{+}\right\rangle,
$$

so that the residual first derivative coupling term for $X=\gamma$ in the $\phi_{i}^{D}$ diabatic representation is zero. For each $R_{\mathrm{OH}}$ and $R_{\mathrm{HF}}$ value, the reference functions are chosen at $\gamma=\pi$. Several calculations are performed varying $\gamma$ continuously from $\pi$ to 0 , using variable steps. Such procedure gives rise to the diabatic curves, $E_{i}^{D}$, shown in Fig. 3.

In Fig. 3 the diabatic energies obtained with the two methods are compared with the adiabatic eigenvalues, at three different nuclear configurations along the reaction path. The two diabatization schemes yield equivalent diabatic energies near collinear OHF, $\gamma \approx \pi$. However, as $\gamma$ decreases the two diabatic schemes become significantly different, both yielding the adiabatic eigenvalues when the diabatic electronic matrix is diagonalized.

Detailed numerical description of the conical intersection at $R_{\mathrm{HF}}=2.0$ a.u. and $R_{\mathrm{OH}}=2.98985$ a.u. is made. The diabatic, $E_{i}^{E}$, and adiabatic, $E_{ \pm}$, energies are shown in the lower panel of Fig. 4. The diabatic energies are nearly constant and degenerate, while the adiabatic eigenvalues change linearly with the angle for a small angular interval around the conical intersection. The mixing angles obtained with the derivative- and energy-based methods, in the middle panel of Fig. 4, are in excellent agreement, showing that near the conical intersections they are nearly equivalent. At lower $\gamma$, the two mixing angles become different, and the corresponding diabatic energies are different, as shown in Fig. 3.

The $\left\langle\varphi_{-}|\partial / \partial \gamma| \varphi_{+}\right\rangle$coupling diverges at conical intersections, and attention should be paid to convergence problems for their numerical obtainment. Thus, a very small step in the angular coordinate $\left(10^{-5}\right.$ degrees) was used. Such small step requires the use of very well converged MRCI wave func-

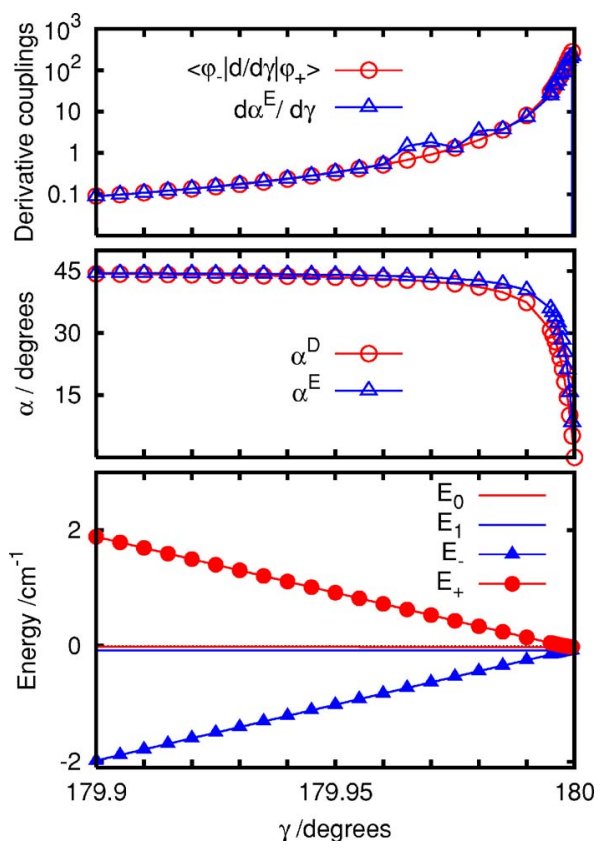

FIG. 4. Bottom panel: Diabatic, $E_{i}^{E}$, and adiabatic, $E_{ \pm}$, energies $\left(\right.$in $\mathrm{cm}^{-1}$ ) as a function of the $\mathrm{OHF}$ angle $\gamma$ calculated at $R_{\mathrm{HF}}=2$ a.u. and $R_{\mathrm{OH}}$ $=2.98985$ a.u., relative to the energy of the crossing. Middle panel: Mixing angles $\alpha^{D, E}$ calculated with the derivative method of Simah et al. (Ref. 43) and the energy-based method of this work, respectively. Top panel: $\left\langle\varphi_{+}|\partial / \partial \gamma| \varphi_{-}\right\rangle$(calculated using the difference finite method of the MOLPRO program) and $\partial \alpha^{E} / \partial \gamma$, both calculated with $\Delta \gamma=10^{-5}$ degrees.

tions, which was achieved by studying the convergence of nonadiabatic couplings when lowering down several convergence thresholds in the MOLPRO package.

On the other side, the calculation of $\partial \alpha^{E} / \partial \gamma$, appearing in Eq. (11), requires also very accurate energies with many significant figures, to be evaluated adequately. Also, $E_{1}$ (the $1^{3} A^{\prime}$ adiabatic eigenvalue) must be degenerate with $E_{-}$or $E_{+}$ at collinear geometries. This is true within the numerical accuracy of the calculations, but there is always a small error, $\approx 0.2 \mathrm{~m} E_{\mathrm{h}}$. In the present energy-based method, for fixed $R_{\mathrm{OH}}$ and $R_{\mathrm{HF}}$ the $E_{1}$ curves are shifted so that they become degenerate with the closest of the $E_{ \pm}$eigenvalues at $\gamma=\pi$.

$\left\langle\varphi_{-}|\partial / \partial \gamma| \varphi_{+}\right\rangle$and $\partial \alpha^{E} / \partial \gamma$ derivative couplings, in Fig. 4, are very similar, and the residual coupling, Eq. (11), becomes negligible in the energy-based diabatization method proposed here. The derivative of the mixing angle in Eq. (12) becomes

$$
\frac{\partial \alpha}{\partial X}=\frac{\left(E_{0}-E_{1}\right) \partial V_{1} / \partial X-V_{1} \partial\left(E_{0}-E_{1}\right) / \partial X}{\left(E_{0}-E_{1}\right)^{2}+4 V_{1}^{2}} .
$$

This formal expression and Eq. (12) are valid for all diabatization methods, being the difference in the obtaining of $E_{0}$, $E_{1}$, and $V_{1}$. In this expression the divergence occurs at the conical intersection, i.e., when $\left(E_{0}-E_{1}\right)=0$ and $V_{1}=0$, as described by the denominator, the same for all $X=\gamma, R_{\mathrm{OH}}, R_{\mathrm{HF}}$. The numerator of this expression is rather small: $E_{0}-E_{1}$ is small and slowly varies with nuclear coordinates, and $V_{1}$ coupling varies as $\sin \gamma$, becoming very small near the conical intersection at $\gamma=\pi$. This term accounts for the singularity appearing in the nonadiabatic derivative couplings in Eq. 

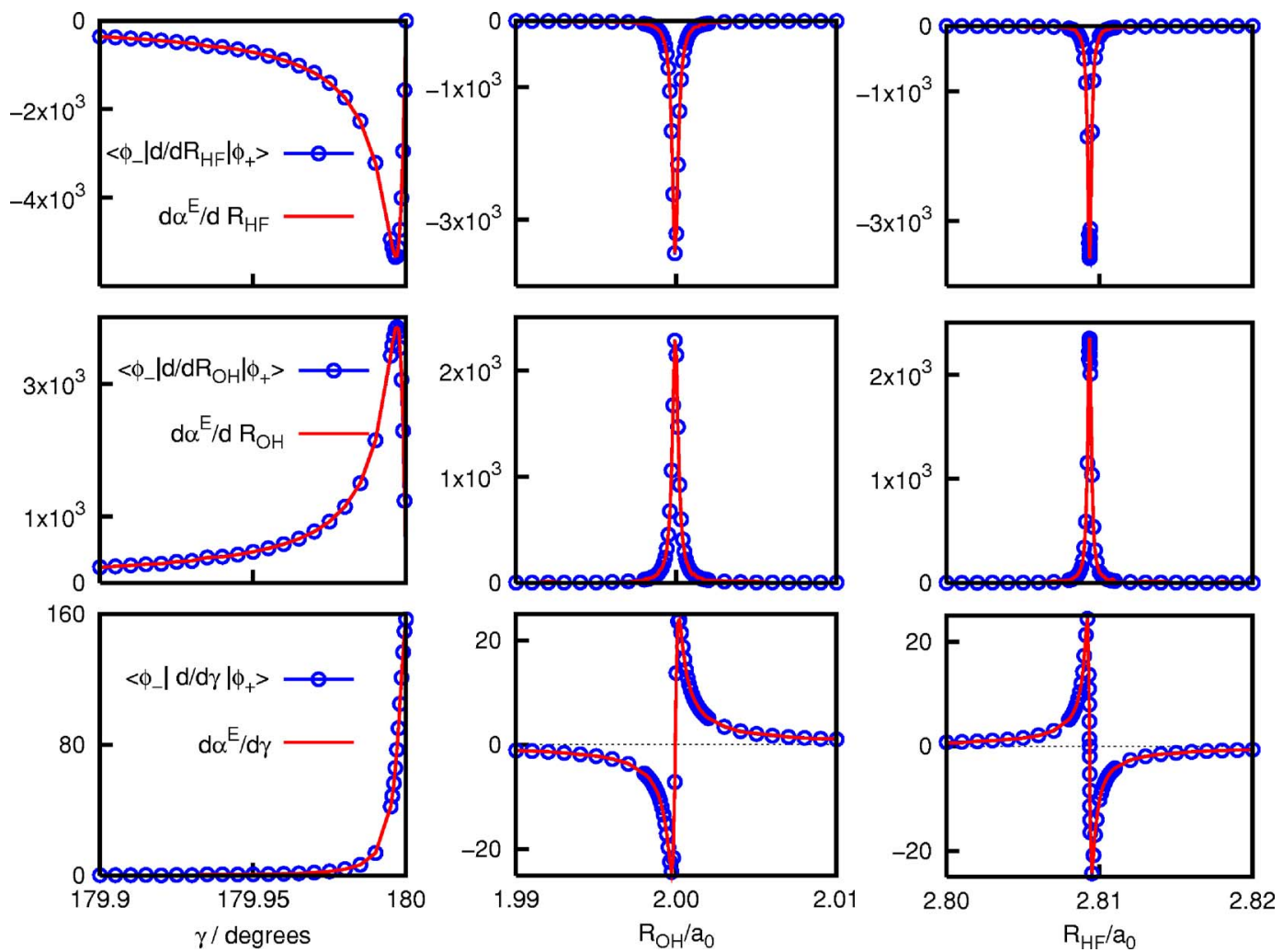

FIG. 5. Nonadiabatic derivative couplings calculated with the CAS method (continuous line) and $\partial \alpha^{E} / \partial X$ (dashed line) for $X=R_{\mathrm{OH}}, R_{\mathrm{HF}}, \gamma$ as a function of the three internal coordinates, keeping the other two near the conical transition at $R_{\mathrm{HF}}=2.8093$ a.u. and $R_{\mathrm{OH}}=2.0000$ a.u., keeping $\gamma=179.99$ to avoid numerical problems.

(1), and its subtraction produces small residual derivative couplings near conical intersections.

To check the connection among the different derivative couplings at conical intersections, here we have also computed $\left\langle\varphi_{-}\left|\partial / \partial R_{\mathrm{OH}}\right| \varphi_{+}\right\rangle$and $\left\langle\varphi_{-}\left|\partial / \partial R_{\mathrm{HF}}\right| \varphi_{+}\right\rangle$. To reduce convergence problems, we have performed the calculations using the complete active space (CAS) method. Also, the calculations are performed at configurations slightly shifted from the conical intersection, to avoid the singularity of nonderivative couplings, allowing more precise numerical results. In Fig. 5 the nonadiabatic derivative couplings calculated in the adiabatic representation are compared with the $\partial \alpha^{E} / \partial X$ obtained in the present method for the different internal coordinates. The two quantities are almost indistinguishable close to the intersections (with an error lower than $1 \%$ ), with the same singularitylike behavior, Eq. (14). This demonstrates that the singularity is essentially associated with the energy crossing as expressed in Eq. (14). When the numerical errors are small the nonadiabatic couplings calculated from the mixing angle obtained from energies, Eq. (12), become exactly the singular term of the nonadiabatic coupling. Due to the connection among the singularities obtained for the derivatives with respect to different internal coordinates, in Eq. (14), when one of them is regularized, the rest become automatically regularized as well. When the numerical errors are larger, as it is the case using MRCI method shown in Fig. 4, there are some disagreements which yield a difference among quantities obtained using different methods.

Therefore, the residual derivative couplings in the result- ing diabatic representation, Eq. (11), do not show the singularity, leading to regularized diabatic states. We can therefore conclude that the method proposed is essentially equivalent to the procedure of Thiel and Köppel ${ }^{40}$ of removing the leading singular derivative coupling terms of the nonadiabatic couplings.

Far from the conical intersections, the two derivative couplings of Fig. 5 present larger relative differences, but small absolute values. Therefore, the residual couplings become different from zero but small, and it would be convenient to evaluate them to analyze their effect. However, the separation of the adiabatic eigenvalues increases far from conical intersections, and therefore such couplings are expected to become more inefficient. Since the two diabatization methods yield the same adiabatic eigenvalues, once the residual couplings become small they become equivalent. In what follows, the residual derivative couplings will be neglected.

The method can be easily generalized to more states when similar conditions are fulfilled. As an example, in the $\mathrm{OH}\left({ }^{2} \Pi\right)+\mathrm{F}\left({ }^{2} P\right) \rightarrow \mathrm{O}\left({ }^{1} D\right)+\mathrm{HF}\left({ }^{1} \Sigma^{+}\right)$reaction there are five electronic states which also cross along the reaction path, as shown in Fig. 1. The diabatic states would have a good projection of the electronic orbital angular momentum, $\Lambda=0$, \pm 1 , and \pm 2 . Performing a symmetry transformation analogous to that of Eq. (7) the electronic Hamiltonian becomes also block diagonal, composed by $2 \times 2$ and $3 \times 3$ matrices. Neglecting the direct couplings among states with $\Delta \Lambda>1$, and assuming that $E_{\Lambda}=-E_{-\Lambda}$, the approximated Hamiltonian 
matrix has five unknown parameters which could be determined from the knowledge of the five adiabatic eigenvalues. Work in that direction is now in progress.

The simplicity and easy generalization of the proposed method present several advantages with respect to derivative-based methods.

- The derivative-based method requires performing the calculations at fixed $R_{\mathrm{OH}}$ and $R_{\mathrm{HF}}$ and varying the angle with a small angular step to follow the change in the reference orbitals and CI matrices. In the present method points for a grid of angles are also needed, but only to correct the lack of degeneracy at collinear geometries (due to numerical inaccuracies of the ab initio method used), which allows larger angular steps.

- The derivative-based method presents many problems when other states cross with the two states of interest, presenting discontinuities in the mixing angle. In the system under study this is very often the case, specially in the $\mathrm{OH}+\mathrm{F}$ channel. To solve this problem, more roots should be calculated.

- Finally, the present method considers the $E_{-1}=E_{1}$ by definition, while the derivative-based method does not. Such degeneracy is consistent with the model and allows the introduction, at least approximately, of coupling Coriolis terms among states belonging to $A^{\prime}$ and $A^{\prime \prime}$ symmetries when the overall rotation of the whole system is considered.

\section{Coupled diabatic potential energy surfaces}

Adiabatic energies have been calculated using the MOLPRO suite of programs ${ }^{44}$ as described in Ref. 25 and will be only briefly described here. The monoelectronic basis set used for the three atoms is the correlation-consistent polarized valence triple zeta basis sets augmented with diffuse functions (aug-cc-pVTZ) of Dunning and co-workers. ${ }^{49,50}$ Molecular orbitals are optimized by a full valence (14 electrons in 9 orbitals) state-averaged complete active space selfconsistent field (SA-CASSCF) calculation. ${ }^{51,52}$ All calculations are performed in $C_{s}$ group of symmetry, and the states included in the state-averaged procedure are $\operatorname{six}{ }^{1} A^{\prime}$, five ${ }^{1} A^{\prime \prime}$, five ${ }^{3} A^{\prime}$, and five ${ }^{3} A^{\prime \prime}$, to obtain a good degeneracy in reactant and product states, as well as for the double degenerated $\Pi, \Delta$ states at collinear geometries. Finally, the adiabatic energies were obtained with an ic-MRCI (Refs. 45 and 46) method, including the Davidson correction. ${ }^{53}$

Initially, the method of Aguado and Paniagua ${ }^{54}$ was used to fit an analytic expression to represent the diabatic matrix in all the configuration space. Such method yields very accurate fits for $E_{1}$ and $E_{0}$. The fit obtained for $E_{1}$, corresponding to the adiabatic ${ }^{3} A^{\prime}$ state, was described in Ref. 25 . The fit of $E_{0}$ obtained here had a rms error lower than $0.05 \mathrm{eV}$. The problem, however, is the fit of $V_{1}$ in Eq. (10): even when rather low errors were obtained, the resulting adiabatic eigenvalues, $E_{ \pm}$obtained using Eq. (9), presented relatively important deviations due to the nonlinear character of the coupling.

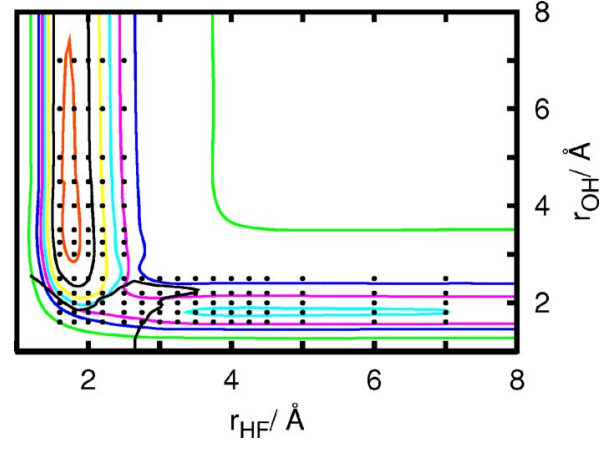

FIG. 6. Radial grid, where $a b$ initio points were calculated, is shown over the two-dimensional PES of ${ }^{3} \Sigma^{-}$state, also showing the intersection seam.

Another alternative method is used, instead, which consists in the following.

(1) The different terms of the diabatic matrix are expanded as a linear combination of normalized associated Legendre functions, ${ }^{55} \mathcal{P}_{\lambda m}(\cos \gamma)$, as

$$
\begin{aligned}
E_{i}\left(R_{\mathrm{OH}}, R_{\mathrm{HF}}, \gamma\right)= & \sum_{\lambda=0}^{\lambda_{\max }} E_{i}^{\lambda}\left(R_{\mathrm{OH}}, R_{\mathrm{HF}}\right) \mathcal{P}_{\lambda 0}(\cos \gamma) \\
& \text { with } i=0,1, \\
V_{1}\left(R_{\mathrm{OH}}, R_{\mathrm{HF}}, \gamma\right)= & \sum_{\lambda=1}^{\lambda_{\max }} V_{1}^{\lambda}\left(R_{\mathrm{OH}}, R_{\mathrm{HF}}\right) \mathcal{P}_{\lambda 1}(\cos \gamma),
\end{aligned}
$$

where $E_{i}^{\lambda}\left(R_{\mathrm{OH}}, R_{\mathrm{HF}}\right)$ and $V_{1}^{\lambda}\left(R_{\mathrm{OH}}, R_{\mathrm{HF}}\right)$ are obtained by Gauss-Legendre integration (with 100 points) for each fixed value of $R_{\mathrm{OH}}$ and $R_{\mathrm{HF}}$. The adiabatic energies are calculated in a nonequidistant angular grid composed by 40 points, very dense for $\gamma \approx \pi$ and have progressively more spaced points as $\gamma \rightarrow 0$. A monodimensional spline interpolation procedure is used to evaluate the energies at the Gauss-Legendre quadrature points. At short angles the adiabatic energies are very high, specially in the $\mathrm{OH}+\mathrm{F}$ channel, and a cutoff of 0.125 a.u. above the $\mathrm{O}+\mathrm{H}+\mathrm{F}$ threshold is introduced. Finally, 100 functions are kept in the expansions of Eq. (15) which are by far enough to reproduce the anisotropy for all the calculated points.

(2) The calculations are performed in a bidimensional radial grid composed by 120 points distributed along the reaction path, as described in Fig. 6. After doing the expansion of Eq. (15), the $E_{i}^{\lambda}\left(R_{\mathrm{OH}}, R_{\mathrm{HF}}\right)$ and $V_{1}^{\lambda}\left(R_{\mathrm{OH}}, R_{\mathrm{HF}}\right)$ are obtained by two-dimensional spline interpolation ${ }^{56}$ in the rectangular region covered by the calculations.

(3) Finally, outside the region of calculated points in Fig. 6, the previous fits for $E_{0}$ and $E_{1}$ are used, while $V_{1}$ is assumed to be zero. The missing points in Fig. 6, corresponding to long $R_{\mathrm{OH}}$ and $R_{\mathrm{HF}}$, were obtained using these analytical fits to generate a complete rectangular grid required for the spline interpolation. The matching between the two regions, the first one covered by the $2 d$ spline interpolation and the second one described by the fit, was carefully done to avoid problems at edges. 
GLOBAL MEP's

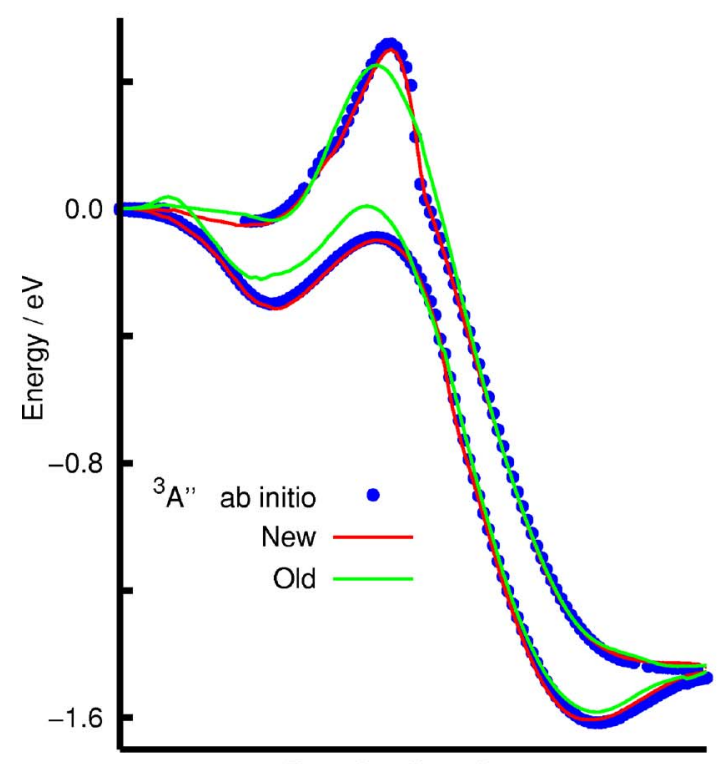

Reaction Coordinate
COLLINEAR MEP's

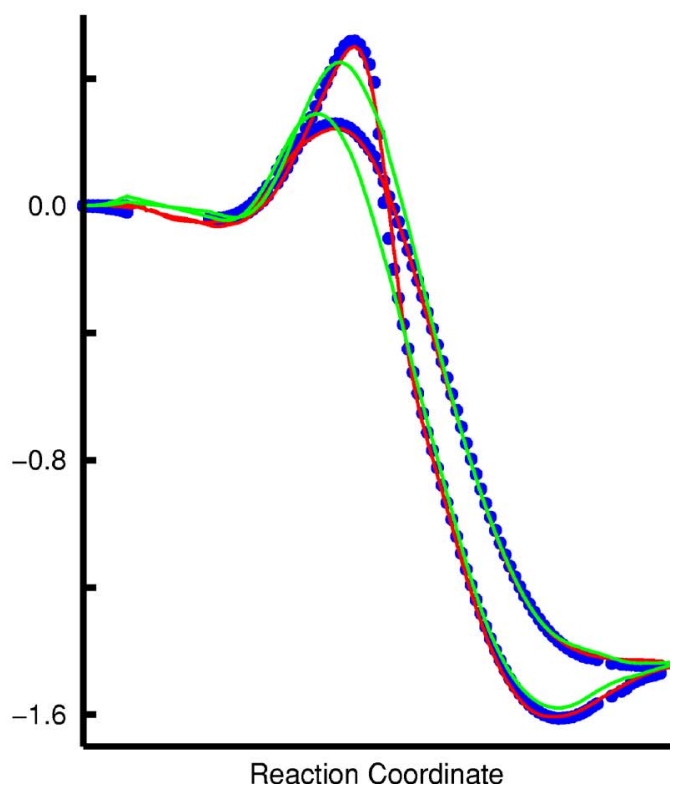

FIG. 7. Minimum energy paths for the three adiabatic eigenvalues obtained from the present coupled DPES, shown in continuous lines. The points correspond to ab initio calculations performed at the corresponding configuration points, and the green line corresponds to the previous APES of Refs. 24 and 25 . Energies are in $\mathrm{eV}$. The left panel corresponds to the three-dimensional case, while the right panel is for collinear configurations.

The minimum energy paths for the three adiabatic eigenvalues $E_{-}, E_{+}$, and $E_{1}$ resulting from the interpolated DPES were obtained and new ab initio calculations were performed along them, shown in Fig. 7 for the two ${ }^{3} A^{\prime \prime}$ APESs. An analogous procedure was followed for collinear configuration (see Fig. 7). The present DPES yields a very accurate description of the points along the whole path. It is notorious that is very well adapted to describe the cusps due to conical intersections along the collinear geometries. Also the height and position of the barrier and wells are very well reproduced.

The adiabatic energies obtained in the previous fits of Refs. 24 and 25 do not show the same agreement with the calculated $a b$ initio points. For the excited PESs we can observe that the interpolation procedure is better in reproducing the cusp due to conical intersections than the previous fit: ${ }^{25}$ in the previous APESs the $a b$ initio points near the cusps were eliminated to avoid problems in the fitting procedure. The present APES also improves the description of the height and position of the barrier for the excited state as compared to the previous fit. ${ }^{25}$

The larger differences found in the barrier height of the $1^{3} A^{\prime \prime}$ ground state are due to the use of a lower number of states in the state-averaged CAS procedure to optimize molecular orbitals, as explained in Ref. 25. The new ab initio points present a significantly lower barrier.

The behaviors of the DPES and APES proposed here at the conical intersections are compared in more detail with $a b$ initio calculations in Fig. 8. The top panels correspond to $\gamma$ $=\pi$, and the diabatic and adiabatic curves are degenerate. The $a b$ initio points, however, show a little shift, more clearly seen in the right panel corresponding to shorter radial interval. Nevertheless, the energy shift is lower than $0.005 \mathrm{eV}$, below the error attributed to the ab initio points.
Also, the position of the crossing point is quite precisely described, with an error lower than 0.02 a.u. of distance.

It is also important to note that in the left panels there is a third state which crosses at $\gamma=\pi$ with the second adiabatic state, giving rise to a new conical intersection at a very close position with respect to the first one described in detail

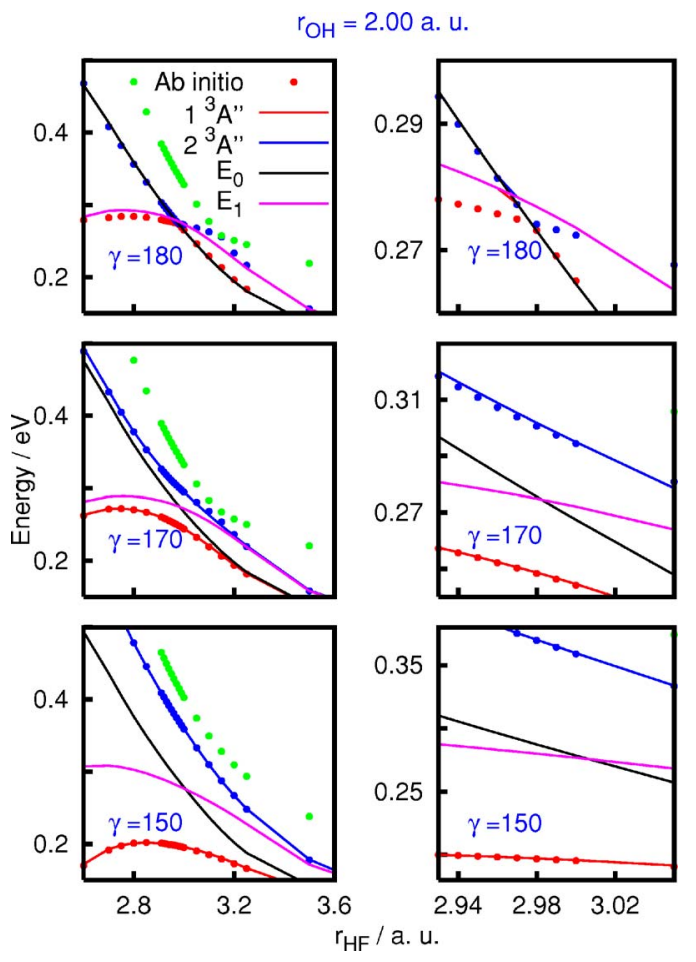

FIG. 8. Diabatic and adiabatic PESs (lines) at conical intersections compared with ab initio calculations (in points) for $R_{\mathrm{OH}}=2$ a.u. and three different angles, $\gamma=180$ (top), 170 (middle), and 150 (bottom). The right panels are insets of the left panels in a shorter interval to show the details of the crossing. 


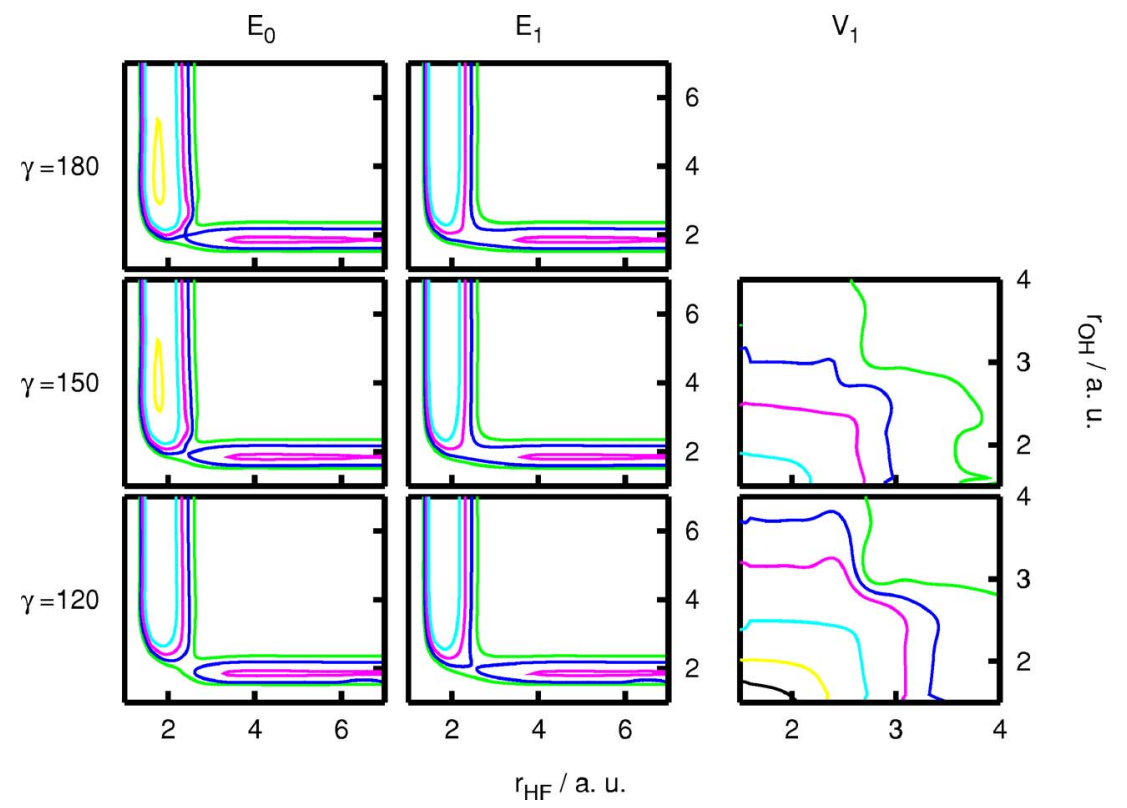

FIG. 9. Contour plots of the interpolated diabatic PES, $E_{0}$ (left panels), $E_{1}$ (middle panels), and $V_{1}$ (right panels) for several angles $\gamma$. The energies are $-1.5,-0.5,0,0.5$, and $1 \mathrm{eV}$ for $E_{i}$ and $1.5,1.0,0.5,0.2,0.1$, and $0.01 \mathrm{eV}$ for $V_{1}$ above. This third state probably correlates with ${ }^{3} \Sigma^{+}$state shown in Fig. 1. This is one example of the complexity related to the use of derivative-based methods, because the mixing angle would show another sharp change. To avoid this problem, a third state should be included. This situation becomes worse as $R_{\mathrm{HF}}$ increases because there are more states crossing, the two ${ }^{3} \Delta$ states in Fig. 1, which makes that in that asymptote there are at least six quasidegenerate triplet states, plus some other states crossing with them at higher energies.

The lower panels of Fig. 8, for $\gamma=170^{\circ}$ and $150^{\circ}$, show a clear difference between diabatic and adiabatic energies. Since the energy intervals in these cases are larger, there is no appreciable difference between the resulting adiabatic states and the new calculated points at the avoided crossings. The largest disagreement found for $\gamma=\pi$ is below the estimated errors of the ab initio points, and we conclude that this description is accurate enough, with an error of $\approx 2 \mathrm{meV}$. Therefore, the present PESs, at diabatic or adiabatic level, are a considerable improvement with respect to the available existing ones.

Finally, the DPESs and couplings are shown in Fig. 9 contour plots as function of the radial coordinates and for several angles.

\section{PHOTOELECTRON DETACHMENT SIMULATIONS}

In a first order perturbative approximation for electric dipole transitions, the differential cross section describing the angular distribution of electrons obtained after photon excitation of a randomly oriented anion in a single initial state, $\Psi_{i}^{J_{i}}$, is given by ${ }^{57-59}$

$$
\frac{d \sigma(h \nu)}{d \Omega}=\sum_{\Lambda_{t} \alpha^{\prime}} \frac{\sigma_{\Lambda_{t} \alpha^{\prime}}(h \nu)}{4 \pi}\left[1+\beta_{\Lambda_{t}} P_{2}(\cos \Theta)\right] .
$$

In this expression, $\theta$ is the angle between the ejected electron velocity vector and the polarization vector of the incident linearly polarized light. $\beta_{\Lambda_{t}}$ is the anisotropy parameter and depends on the electric dipole transition matrix elements between the initial and final states and determines the shape of the angular distributions of the ejected electrons. $\sigma_{\Lambda_{t} \alpha^{\prime}}$ is the partial cross section to form nuclear products in state $\alpha^{\prime}$, after absorption to the electronic state $\Lambda_{t}$, with $\Lambda_{t}$ referring to the projection of the total electronic orbital angular momentum on the body-fixed $z$ axis.

The ejected electron is much faster than the subsequent rearrangement of the neutral molecular system, which evolves slowly in several coupled surfaces towards reactants or products. Thus, the process can be treated approximately as two separated processes: the electron ejection, while nucleus can be considered to stay at rest, and the subsequent nuclear rearrangement which is analyzed by detecting products.

It may be assumed that the electron departs with a single kinetic energy, obtained by the conservation of total energy as $\epsilon=h \nu+E_{\mathrm{OHF}^{-}}-E_{\mathrm{OHF}}$, and does not affect either the reaction dynamics of the nuclear fragments. The electron is not treated explicitly and some approximation is needed. Considering the axial recoil approximation for fast ionization processes, for parallel transitions $\left(\Lambda_{t}=\Lambda_{i}\right)$ it will be considered that $\beta_{\Lambda_{t}}=2$, while for perpendicular transitions $\left(\Lambda_{t}=\Lambda_{i} \pm 1\right)$ $\beta_{\Lambda_{t}}=-1$. Thus, electrons arising from parallel transitions give rise to a distribution proportional to $\cos ^{2} \theta$, while for perpendicular transitions the distributions are proportional to $\sin ^{2} \theta$. Such situation allows the separation of transitions to two different $\Lambda_{t}$ states by simply detecting the electrons at $\Theta=0$ or $\pi / 2$.

$\Lambda_{t}=\Lambda+\lambda$, with $\Lambda$ and $\lambda$ being the electronic orbital angular momentum projections of the neutral OHF fragment and the departing electron, respectively. Since $\lambda$ is not known and it is not detected experimentally, we shall choose its value to reproduce the experimental spectra. In a previous work, ${ }^{26}$ the norm of the transition dipole moments was estimated to establish the relative absorption intensity towards triplet or singlet states. Here, we shall study the directions of the electric dipole transition moments for the triplet states. 
TABLE I. Rovibrational energy levels of the $\mathrm{OHF}^{-}\left({ }^{2} \Pi\right)$ anion for $J_{i}^{\epsilon}=0^{ \pm}$and $1^{ \pm}$. The major helicity component, $\Omega$, is shown for $J=1$. The two cases considered, $\Lambda_{i}=0$ and 1 , are compared. Energies (in $\mathrm{cm}^{-1}$ ) referred to the minimum of the well.

\begin{tabular}{ccccccc}
\hline \hline & $\Lambda_{i}=0$ & & & & $\Lambda_{i}=1$ \\
\cline { 1 - 2 } \cline { 5 - 6 }$J_{i}^{\epsilon}=0^{+}$ & $J_{i}^{\epsilon}=1^{-}$ & & & & & $J_{i}^{\epsilon}=0^{ \pm}$ \\
2261.31 & 2261.98 & & & & 2261.66 & 1 \\
2758.89 & 2759.55 & 0 & & & 2759.22 & 1 \\
3250.51 & 3251.17 & 0 & & & 3250.84 & 1 \\
& 3293.61 & 1 & & 3293.25 & 3293.96 & 0 \\
3767.96 & 3768.62 & 0 & & & 0 \\
& 3793.36 & 1 & 3793.01 & & 0 \\
\hline \hline
\end{tabular}

Once $\beta_{\Lambda_{t}}$ is parametrized, the angular resolved spectra intensity, in Eq. (16), is completely determined by the cross sections $\sigma_{\Lambda, \alpha^{\prime}}$, which depend on the selection rules for electronic transitions, and the Franck-Condon factors due to the amplitude of the nuclear part of the wave functions in the different electronic states involved. Their calculations are described below. Later the results obtained in the adiabatic and diabatic representations will be described.

\section{A. Simulation of absorption intensities}

The total absorption cross section from the anion state $\Psi_{i}^{J_{i}}$ (with an electronic orbital angular momentum projection $\Lambda_{i}$ ) to a final electronic state $\Lambda_{t}$ of the $\mathrm{OHF}+e^{-}$system is expressed in a time dependent treatment as

$$
\begin{aligned}
\sigma_{\Lambda_{t}}(h \nu) & =\sum_{\alpha^{\prime}} \sigma_{\Lambda_{t} \alpha^{\prime}}(h \nu) \\
& =\frac{1}{\pi \hbar} \mathcal{R} e \int_{0}^{\infty} d t e^{-i E t / \hbar}\left\langle\Psi_{\Lambda_{t}}^{J M}(t) \mid \Psi_{\Lambda_{t}}^{J M}(t=0)\right\rangle,
\end{aligned}
$$

where $J$ and $M$ are the total angular momentum and its projection in a space-fixed frame.

The wave packet is propagated in the Hamiltonian corresponding to the OHF neutral system. The reaction dynamics yields preferentially $\mathrm{HF}\left({ }^{1} \Sigma^{+}\right)+\mathrm{O}\left({ }^{3} P\right)$ products, see Fig. 1 , so that the system can be treated as a closed shell molecule plus an open shell atom. The Hamiltonian used is that described by Rebentrost and Lester ${ }^{60}$ in Jacobi coodinates: $\mathbf{r}$, the HF internuclear vector, and $\mathbf{R}$, the vector going from the $\mathrm{HF}$ center of mass to the $\mathrm{O}$ atom, with $\gamma$ being the angle between them. A body-fixed frame is defined by three Eulerian angles $(\phi, \theta, \chi)$, with the three atoms in the $x$ - $z$ plane and the $z$ axis pointing along the $\mathbf{R}$ vector. The body-fixed frame used is not equal to that defined for the electronic Hamiltonian, in which the $z$ axis coincides with the OF internuclear axis. However, since hydrogen is very light and always attached to one of the two heavier atoms, it can be considered that $\mathbf{R}_{\mathrm{OF}}$ is approximately parallel to $\mathbf{R}$.

The wave packet is then represented as ${ }^{61-63}$

$$
\Psi_{\Lambda_{t}}^{J M \epsilon}=\sum_{\Omega, \Lambda^{\prime}, j} \frac{\Phi_{\Omega, \Lambda^{\prime}, j}^{J M \Lambda_{t}}(r, R)}{r R} W_{\Omega \Lambda^{\prime}}^{J M \epsilon},
$$

where the angular functions are eigensolutions of the inversion of spatial coordinates (with eigenvalue $\epsilon$ ) and are defined as

$$
\begin{aligned}
W_{\Omega \Lambda^{\prime}}^{J M \epsilon}= & \sqrt{\frac{2 J+1}{16 \pi^{2}\left(1+\delta_{\Omega \Lambda^{\prime}, 0}\right)}}\left[\mathcal{P}_{j \Omega-\xi}(\gamma) D_{M \Omega}^{J^{*}}(\phi, \theta, \chi)\left|\Lambda^{\prime}\right\rangle\right. \\
& \left.+\epsilon S_{\Lambda^{\prime}}(-1)^{J} \mathcal{P}_{j \xi-\Omega}(\gamma) D_{M-\Omega}^{J *}(\phi, \theta, \chi)\left|-\Lambda^{\prime}\right\rangle\right], \quad(19)
\end{aligned}
$$

where $s_{\Lambda}$ is the parity of the electronic wave function under reflection through the $x-z$ body-fixed plane. In the diabatic representation it takes the values $s_{\Lambda}=+1,-1$, and +1 for $\mathrm{OHF}^{-}\left({ }^{2} \Pi\right), \operatorname{OHF}\left({ }^{3} \Sigma^{-}\right)$, and $\operatorname{OHF}\left({ }^{3} \Pi\right)$, respectively.

In Eq. (19) the $\xi$ quantum number takes different values depending on the electronic representation chosen. Thus, in the diabatic basis, $\xi \equiv \Lambda^{\prime}$, and $s_{\Lambda}=-1$ or +1 for ${ }^{3} \Sigma^{-}$and ${ }^{3} \Pi$, respectively. However, in the adiabatic representation the projection of the orbital angular momentum is not well defined: this is equivalent to using $\xi=0$ and $\Lambda=0$ (and $s_{\Lambda}$ $=+1)$ in Eq. (19).

The details of the wave packet propagation are described in detail in Refs. 64 and 26. Since the experimental resolution is of $8 \mathrm{meV}$, the autocorrelation functions, in Eq. (17), are multiplied by a $e^{-\Gamma t / \hbar}$ exponential function, with $\Gamma$ $=8 \mathrm{meV}$. Thus most of the resonant structures described before $^{26,64}$ disappear in the spectra, as will be shown in what follows.

\section{B. Initial wave packet}

The initial rovibrational state of the $\mathrm{OHF}^{-}\left({ }^{2} \Pi\right)$ anion, $\Psi_{i}^{J_{i} M_{i}}$, is also expanded according to Eq. (18), considering $\xi=0$ in the adiabatic representation and $\xi= \pm 1$ in the diabatic representation. The three-dimensional PES used corresponds to the $\operatorname{OHF}^{-}\left(X^{2} A^{\prime}\right)$ previously reported ${ }^{64}$ and correlates with a ${ }^{2} \Pi$ doubly degenerate state at collinear geometry. This PES for the anion is in very good agreement with recently published curves for the collinear geometry. ${ }^{65}$ Renner-Teller couplings are neglected, since the first excited ${ }^{2} A$ " state showed a small splitting of $\approx 300 \mathrm{~cm}^{-1}{ }^{64}$ The rovibrational levels for $J=0$ and 1 for the two cases considered, $\Lambda_{i}=0$ (adiabatic representation) and 1 (diabatic representation), are listed in Table I. For $\Lambda_{i}=0$, the ground level appears for $J=0$ and 
$\Omega=0$, while for $\Lambda_{i}=1$ it is for $J=1$ and $\Omega=1$. Since the potential well presents a collinear geometry and $\mathcal{P}_{j, \Omega-\Lambda_{i}}(\gamma$ $\rightarrow 0) \propto \sin ^{\Omega-\Lambda_{i}} \gamma$, the lowest eigenvalues correspond to nearly pure $\Omega-\Lambda_{i}=0$ components.

The initial wave packet is constructed by applying the electric dipole transition operator to the $\mathrm{OHF}^{-}$rovibrational state, for each final electronic state $\Lambda_{t}$ of the $\mathrm{OHF}+1 e^{-}$system, as described previously. ${ }^{26}$ In that work a detailed model was built to estimate the relative absorption intensity among the different triplet (three) and singlet (six) states considered in that work. It consisted in using the same molecular orbitals for describing $\mathrm{OHF}^{-}$anion and $\mathrm{OHF}$ neutral systems and comparing the main electronic configurations obtained for the different electronic states of the OHF and $\mathrm{OHF}^{-}$: those configurations of the OHF neutral states contained in the $\mathrm{OHF}^{-}\left({ }^{2} \Pi_{x, y}\right)$ state would correspond to single excitation, thus contributing to the transition dipole moment, while the escaping electron should correspond to a dissociative state describing the ionization. The problem, however, is to determine the helicity $\lambda$ of the escaping electron. To simplify, in Ref. 26, it was assumed that the transition dipole moment $\mathbf{d}$ is isotropic, which is a reasonable assumption for the simulation of integrated cross sections. For the obtainment of the angular resolved spectra, however, it is important to properly define $\lambda$, since it will determine the parallel/perpendicular transition character of the absorption. Since this quantity is unknown, in this work two different cases will be considered:

- $\lambda=0, e(\sigma)$, leading to parallel $\left(\mathbf{d} \equiv \mathbf{d}_{z}\right)$,

$$
\mathrm{OHF}^{-}\left({ }^{2} \Pi\right)+h \nu \rightarrow \operatorname{OHF}\left({ }^{3} \Pi_{x, y}\right)+e(\sigma),
$$

and perpendicular transitions $\left(\mathbf{d} \equiv \mathbf{d}_{x, y}\right)$,

$$
\mathrm{OHF}^{-}\left({ }^{2} \Pi\right)+h \nu \rightarrow \mathrm{OHF}\left({ }^{3} \Sigma^{-}\right)+e(\sigma) \text {; }
$$

- $\lambda= \pm 1, e(\pi)$, leading to parallel $\left(\mathbf{d} \equiv \mathbf{d}_{z}\right)$,

$$
\mathrm{OHF}^{-}\left({ }^{2} \Pi\right)+h \nu \rightarrow \mathrm{OHF}\left({ }^{3} \Sigma^{-}\right)+e(\pi),
$$

and perpendicular transitions $\left(\mathbf{d} \equiv \mathbf{d}_{x, y}\right)$,

$$
\mathrm{OHF}^{-}\left({ }^{2} \Pi\right)+h \nu \rightarrow \operatorname{OHF}\left({ }^{3} \Pi_{x, y}\right)+e(\pi) \text {. }
$$

\section{Angular resolved cross section: Adiabatic representation}

Triplet states are the only ones contributing to the spectrum for electron kinetic energies higher than $1 \mathrm{eV}$, at the wavelength of $213 \mathrm{~nm}$. For lower energies, the singlet states contribute significantly, as demonstrated in Ref. 26 using two-dimensional potential energy surfaces. Attention will then be focused on the spectrum structures appearing for $\epsilon$ $>0.8 \mathrm{eV}$.

The spectra obtained in the adiabatic representation for the $\mathrm{OHF}^{-}\left({ }^{2} \Pi, J=0\right) \rightarrow \operatorname{OHF}\left(1^{3} A^{\prime \prime}, 2^{3} A^{\prime \prime}\right.$, and $\left.1^{3} A^{\prime}, J=1\right)$ $+1 e(\lambda)$ transitions are shown in Fig. 10. These spectra were obtained as a function of the $E_{\mathrm{OHF}}$ energy and then expressed in terms of the electron kinetic energy, using $\epsilon=2.44 \mathrm{eV}$ $-E_{\mathrm{OHF}}$, to simulate the experimental data at $213 \mathrm{~nm}(2.44$ corresponds to the photon energy plus the dissociation en-

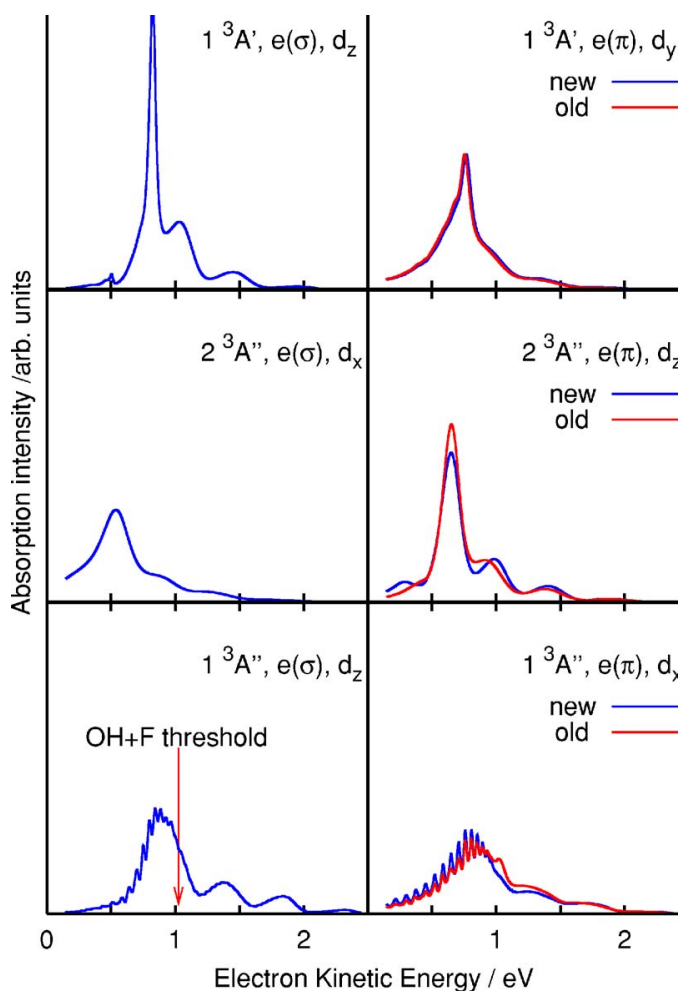

FIG. 10. Individual absorption spectra for each adiabatic electronic state of neutral OHF system. The $J=1^{-} \leftarrow J_{i}=0^{+}$transition and how the components of the electric dipole moment depends on the $\sigma$ or $\pi$ character of the departing electron are considered. In all panels the APES used is that described in this work. In the right panels results obtained with the old APESs of Refs. 24 and 25 are also shown for comparison.

ergy of the anion). The wave packet mostly evolves towards the first $\mathrm{O}\left({ }^{3} P\right)+\mathrm{HF}$ threshold, open at all electron kinetic energies considered. The $\mathrm{F}+\mathrm{OH}(v=0)$ rearrangement channel opens for electron kinetic energies below the arrow indicated in the bottom left panel. All the spectra show a series of broad peaks, which are associated with HF vibrations, as described previously. ${ }^{22,23,64}$

The two excited states do not present any well, thus yielding broad structures and there are only some traces of resonances for the ground $1{ }^{3} A^{\prime \prime}$ state, which presents two wells, one in each rearrangement channel. These resonances are above the $\mathrm{F}+\mathrm{OH}(v=0)$ threshold and have shorter lifetimes because the opening of the reaction threshold increases significantly the density of final states. The resonances below this threshold are very narrow and correspond to quasibound states sitting on the linear $\mathrm{HF}+\mathrm{O}$ well, which dissociates through rotational and/or vibrational predissociation. They disappear when only considering short time dynamics, consistent with the $8 \mathrm{meV}$ of resolution.

In the right panels of Fig. 10, the spectra obtained with the present APESs are compared with the old APESs. ${ }^{24,25}$ Only small changes are appreciable for the two ${ }^{3} A^{\prime \prime}$ states, which are not very significant.

It is important to note, however, the large difference obtained when considering $e(\sigma)$ (left panels) and $e(\pi)$ (right panels) escaping electrons. The component of the transition dipole moment $\mathbf{d}$ involved in the absorption changes with the helicity of the escaping electron. The initial state, $\Psi_{i}^{J_{i}=0}$, in 

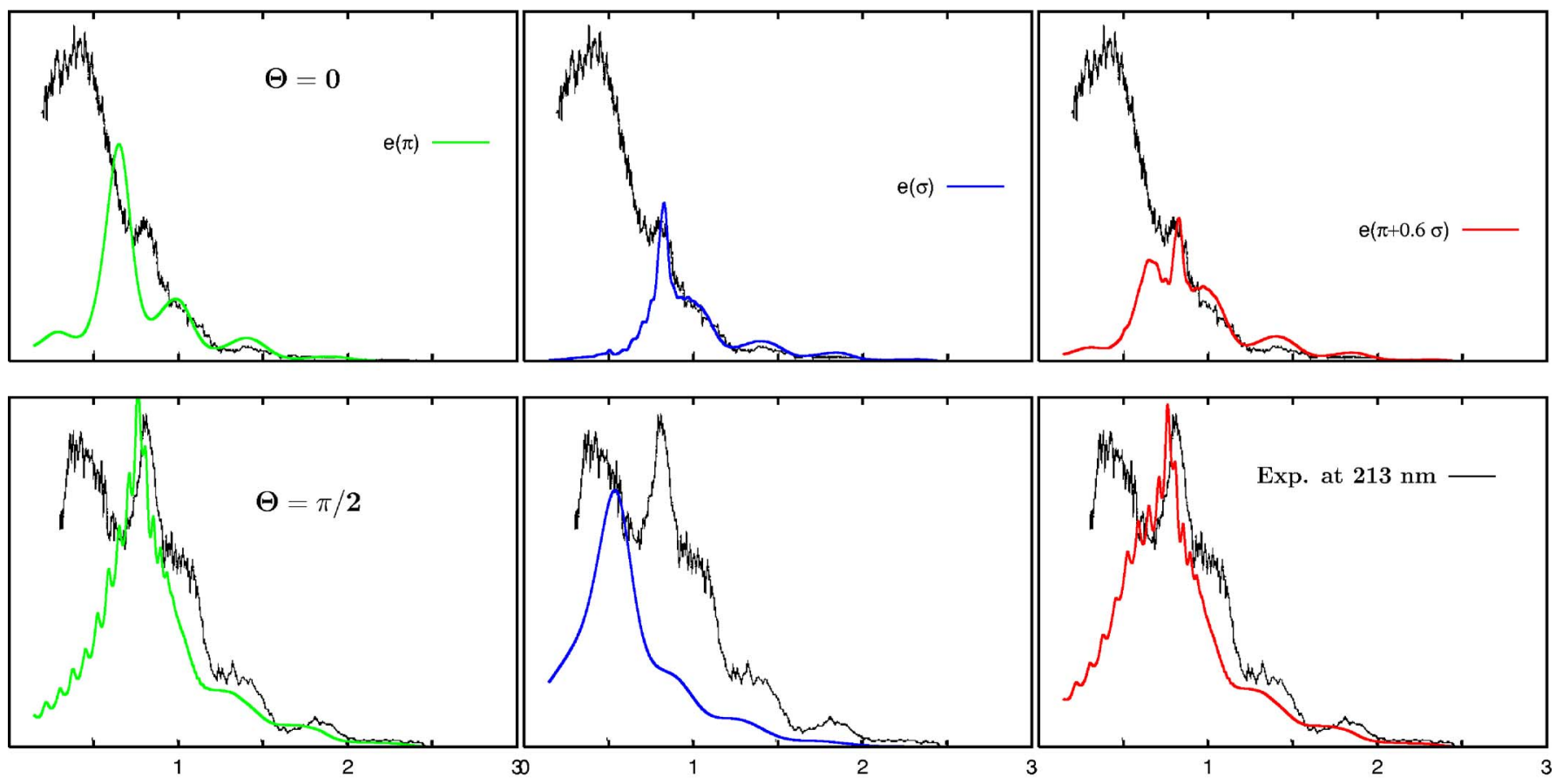

Electron Kinetic Energy / eV

FIG. 11. Angular resolved spectra for ejected electrons at $\Theta=0$ and $\pi / 2$ corresponding to the new adiabatic triplet states. Three possibilities are considered for the projection of the orbital angular momentum of the ejected electron: pure $\sigma$, pure $\pi$, and a linear combination of $\sigma$ and $\pi$.

the adiabatic representation corresponds to $\Omega=0$, and perpendicular transitions, $d_{x}$ or $d_{y}$, will yield preferentially $\Omega$ $= \pm 1$ components in the neutral fragments, while parallel transitions will not change the helicity of the system. The initial wave packet created is expanded as a superposition of spherical harmonics, which behaves as $Y_{j \Omega}(\gamma) \propto \sin ^{\Omega} \gamma$ when $\gamma \rightarrow 0$ or $\pi$. Thus, initial wave packets with only $\Omega=1 \mathrm{com}$ ponents do not explore collinear configurations, while those with $\Omega=0$ components can. This fact accounts for the large difference found among left/right panels in Fig. 10.

The angular resolved total spectra recorded at $213 \mathrm{~nm}$ for $\Theta=0$ and $\pi / 2$ (Refs. 31 and 32) are simulated summing over the contributing electronic transitions, depending on the $\sigma$ or $\pi$ nature of the ejected electron. If the escaping electron is purely $\sigma$ (or $\pi$ ), a parallel transition would excite the ${ }^{3} \Pi_{x, y}$ (or ${ }^{3} \Sigma^{-}$) states, while a perpendicular transition would excite the ${ }^{3} \Sigma^{-}$(or ${ }^{3} \Pi_{x, y}$ ) state. The ${ }^{3} \Pi_{y}$ state corresponds to the adiabatic $1{ }^{3} A^{\prime}$, while the ${ }^{3} \Sigma^{-}$and ${ }^{3} \Pi_{x, y}$ states mix in the adiabatic representation to yield the two ${ }^{3} A^{\prime \prime}$ states and they have to be assigned: at the linear equilibrium geometry of the $\mathrm{OHF}^{-}$anion, $1^{3} A^{\prime \prime} \equiv{ }^{3} \Pi_{x}$ and $2^{3} A^{\prime \prime} \equiv{ }^{3} \Sigma^{-}$, as can be seen in Fig. 1. With this assignment, the spectra are conveniently combined and can be directly compared with the experimental spectra, as shown in Fig. 11.

The parallel spectrum, $\Theta=0$, for $e(\sigma)$ (obtained summing $1^{3} A^{\prime \prime}$ and $1^{3} A^{\prime}$ contributions) is rather well reproduced for electron kinetic energies above $0.75 \mathrm{eV}$ (below which the singlets also contribute). The perpendicular spectrum, $\Theta=\pi$, corresponding to pure $2^{3} A^{\prime \prime}$, does not reproduce the different structures, specially for energies higher than $2 \mathrm{eV}$ and the experimental peak appearing at $\approx 0.8 \mathrm{eV}$.

For $e(\pi)$, however, the situation is the reverse: the perpendicular spectrum is reproduced reasonably well, while the parallel one shifts the experimental peak (at $\approx 0.8 \mathrm{eV}$ ) toward lower energies. The assumption of pure $e(\pi)$ contributions is, nevertheless, better than $e(\sigma)$.

One could also assume that the escaping electron may arise with $\lambda=0$ or 1 , giving rise to two contributions per state. Such assumption is somehow equivalent to considering that transition dipole moment depends on the bending angle $\gamma$, while here it has been considered to be a constant as a function of all internal coordinates. To check this assumption, in Fig. 11 a combination of $e(\pi)+0.6 e(\sigma)$ is obtained as an optimal linear combination describing most of the features of the experimental spectra.

One of the problems of this adiabatic representation is the assignment of a particular $\Lambda$ character to the electronic states. In spite of the good description with the character assigned at the equilibrium geometry of $\mathrm{OHF}^{-}$, the initial wave packet is placed on the conical intersection seams, as can be seen in Fig. 2, showing that in the adiabatic representation a single $\Lambda$ character cannot be attributed to any of the two ${ }^{3} A^{\prime \prime}$ adiabatic states. It is therefore necessary to improve such situation by using a diabatic representation as it is described below.

\section{Angular resolved cross section: Diabatic representation}

In the diabatic representation the projection of the electronic angular momentum of OHF on the $z$ axis, $\Lambda$, for each electronic state is well defined. For consistency, the $\Lambda_{i}=1$ corresponding to the initial $\mathrm{OHF}^{-}\left({ }^{2} \Pi\right)$ is included also in the calculations. Thus, the ground rovibrational state corresponds to $J_{i}=1$ (instead of 0 , as considered in the adiabatic 


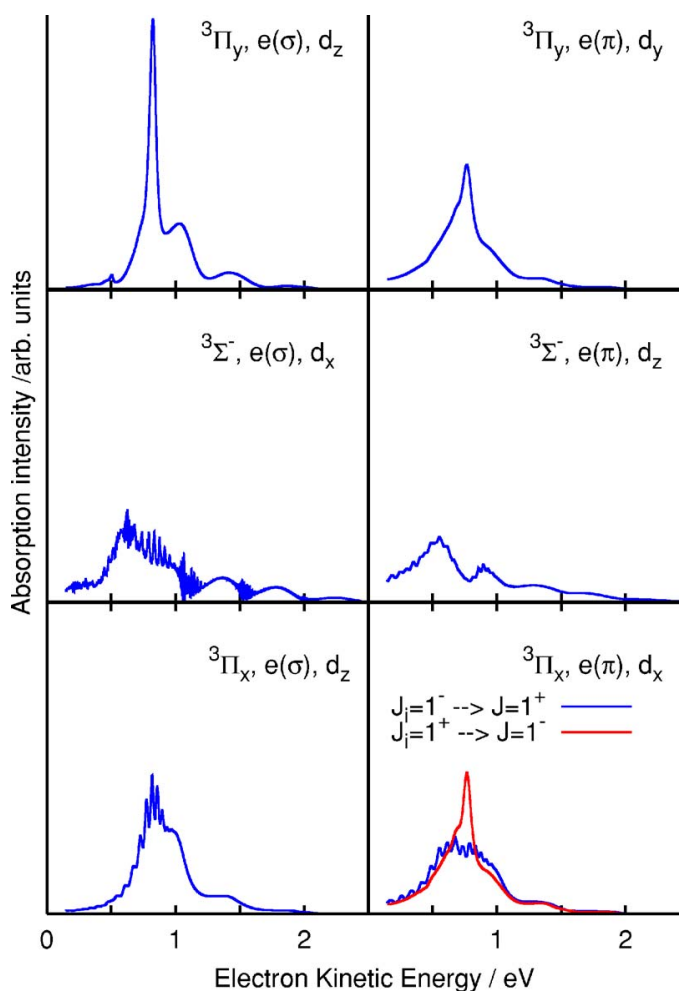

FIG. 12. Individual absorption spectra obtained for the diabatic states, in which the ${ }^{3} \Pi_{x}$ and ${ }^{3} \Sigma^{-}$are coupled, while ${ }^{3} \Pi_{y}$ is considered uncoupled. Each panel shows the results for the $J_{i}=1^{-} \rightarrow J=1^{+}$. The results for the $J_{i}$ $=1^{+} \rightarrow J=1^{-}$are identical except for the ${ }^{3} \Sigma^{-}, d_{x}$, which presents zero amplitude, and for the ${ }^{3} \Pi_{x}, d_{x}$, which is also shown in the bottom right panel.

representation), as can be seen in Table I. In this case the $J_{i}=1 \rightarrow J=1$ rotational transition is simulated, and the calculated spectra are shown in Fig. 12.

The consideration of the $\xi \neq 0$ in Eq. (19) makes that the angular basis set in the diabatic representation is different from that considered in the adiabatic one, which is specially significant at collinear geometries. However, this change is simultaneous for $\mathrm{OHF}^{-}\left({ }^{2} \Pi\right)$ and $\operatorname{OHF}\left({ }^{2} \Pi_{x, y}\right)$, which makes the two representations nearly identical at this respect. Thus, the spectra corresponding to final ${ }^{3} \Pi_{y}$ and $1^{3} A^{\prime}$ are nearly identical, since only one electronic state is considered in both representations. Also, the spectra for ${ }^{3} \Pi_{x}$ are very similar to that obtained for the adiabatic $1^{3} A^{\prime \prime}$ state, the small differences being attributed to the coupling to the ${ }^{3} \Sigma^{-}$state in the diabatic representation. The major changes correspond to the ${ }^{3} \Sigma^{-}$case, in which case $\xi=\Lambda=0$ is different in the initial and final states, producing a significant change in the absorption intensity when comparing diabatic versus adiabatic representations.

In the adiabatic representation, there are resonances only in the $1^{3} A^{\prime \prime}$ state, which is the only one presenting a well in each rearrangement channel and the lower reaction barrier. In the diabatic case, however, the two coupled states present resonant structures, clearly due to the existence of coupling between them. The diabatic ${ }^{3} \Sigma^{-}$state presents the two wells, while the diabatic ${ }^{3} \Pi_{x}$ state has the lowest barrier. Thus, by analogy with the adiabatic states, it is expected that the resonances in the diabatic representation would present larger amplitudes in the wells of the ${ }^{3} \Sigma^{-}$and on the barrier of the ${ }^{3} \Pi_{x}$. Since these resonances have important effects on the reaction dynamics, ${ }^{24,25}$ a more detailed analysis of them is required.

Another change between the spectra obtained in the adiabatic and diabatic representations is the relative intensity between the peaks placed at higher electron kinetic energies, above $1.2 \mathrm{eV}$, and at lower energies. Such differences arise from the curve crossing occurring: the lower (higher) adiabatic curve follows the lower (higher) ${ }^{3} A^{\prime \prime}$ eigenvalue along all the reaction path, while the diabatic curves cross as shown in Fig. 1 for collinear geometries.

Moreover, the allowed transitions change when considering explicitly the electronic orbital angular momentum $\Lambda$ in the angular functions. In the adiabatic representation, $\xi$ $=\Lambda_{i}=\Lambda=0$ and the basis set functions with $\Omega=0$ only exist when $\epsilon=(-1)^{J}$, so that for the initial $\mathrm{OHF}^{-}$there is only one parity state.

In the diabatic representation, however, when $\Lambda \neq 0$ the situation changes, because the two parities exist even when $\Omega=0$. Thus the ground energy level obtained for $J_{i}=1$ is doubly degenerate, each state corresponding to a parity, denoted by $1^{+}$or $1^{-}$hereafter. Thus the number of transitions

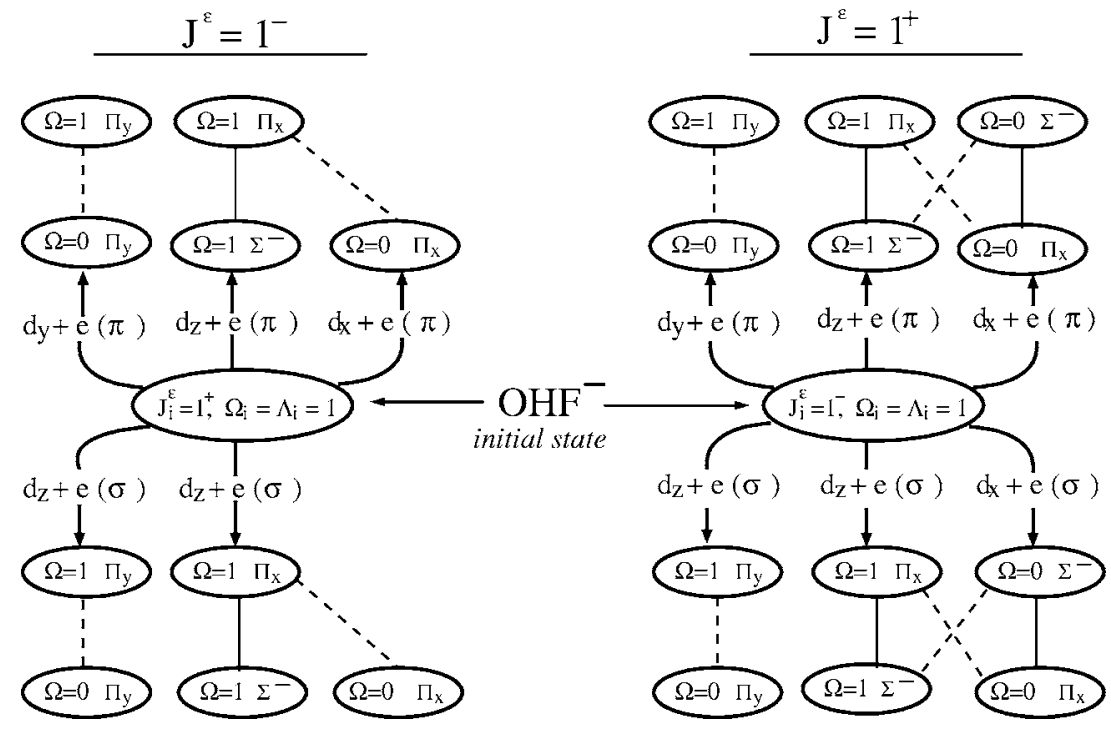

FIG. 13. Electronic transition diagram for the diabatic representation among the different angular basis functions considered. 

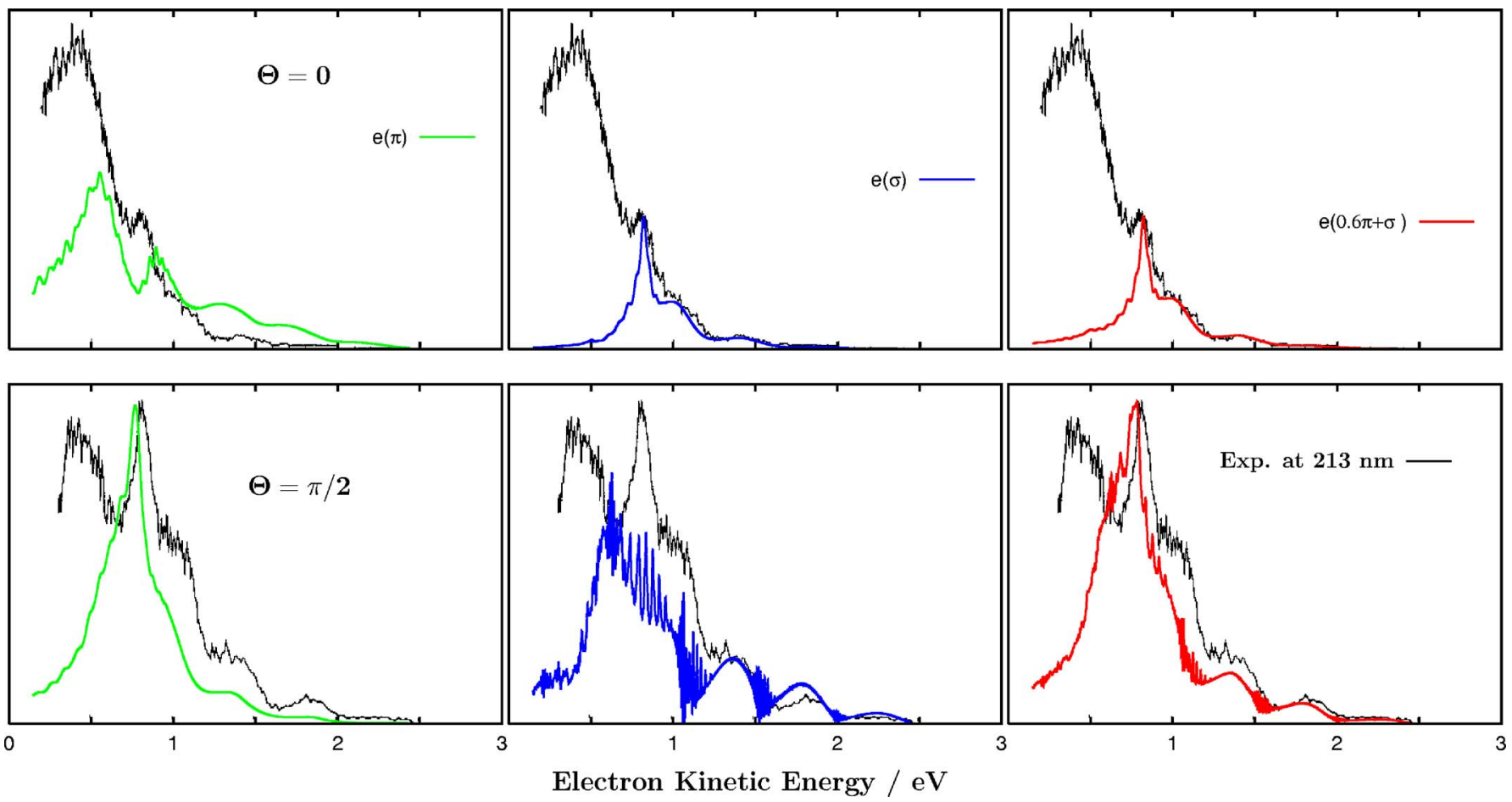

FIG. 14. Same as Fig. 11 but using the DPES as in Fig. 12.

may duplicate. For the neutral OHF system, the same situation occurs for ${ }^{3} \Pi$ electronic states. However, for ${ }^{3} \Sigma^{-}, \Omega$ $=0$ states only exist when $\epsilon=s_{\Sigma^{-}}(-1)^{J}=-(-1)^{J}$. Thus, there will be two kinds of transitions, $1^{-} \rightarrow 1^{+}$(described above and shown in Fig. 12 in solid blue lines) and $1^{+} \rightarrow 1^{-}$. The angular basis set levels appearing for each parity are displayed in Fig. 13, together with the available transitions. Thus, the transitions towards the ${ }^{3} \Pi_{y}$ state are exactly equivalent for the two parity transitions considered. It should be noted that the parallel transition toward the ${ }^{3} \Sigma^{-}$and the ${ }^{3} \Pi_{x}$ state are also equivalent for the two parity cases considered because the Coriolis is negligible and the same functions are involved coupled through the $V_{12}$ diabatic coupling. However, it is important to note that the disappearance of the ${ }^{3} \Sigma^{-}, \Omega=0$ functions for the $J^{\epsilon}=1^{-}$case makes that the perpendicular $1^{+} \rightarrow 1^{-}$transition towards the ${ }^{3} \Sigma^{-}$disappears and for the ${ }^{3} \Pi_{x}$ varies a lot, as shown in the bottom right panel of Fig. 12.

Three different cases have been considered to build the angular resolved spectra in Fig. 14. As in the adiabatic case, assuming pure $\sigma$ or $\pi$ departing electrons cannot describe the two spectra simultaneously. It is then necessary to consider two families of transitions, producing either $\sigma$ or $\pi$ electrons, in order to simulate simultaneously the spectra recorded at these two angles, as shown in Fig. 14. The relative weight of the two characters $(\sigma+0.6 \pi)$ is determined by fitting the experimental spectra and differs from that obtained in the adiabatic representation, mainly because some more transitions occur in the diabatic representation.

As discussed above, there are significant differences among the spectra simulated in the diabatic and adiabatic representations. However, the fitting procedure used to get the relative $\sigma / \pi$ character ratio of the departing electron in the two cases yields results of equivalent quality. This indicates that these spectra are not very sensitive to the nonadiabatic couplings among different electronic states. Some more detailed quantities are required to get information of the nonadiabatic coupling effects on the reaction dynamics, such as the final energy distribution of fragments recently obtained by Deyerl and Continetti ${ }^{33}$ and Continetti, ${ }^{34}$ in coincidence detection experiments of electron and neutral fragments.

The simulated spectra are in rather good agreement with the experimental ones, reproducing all the structures for kinetic energies above $0.75 \mathrm{eV}$. For lower energies the singlet states have to be considered ${ }^{26}$ and some work in this direction is now in progress. The small differences obtained for electron kinetic energies higher than $0.75 \mathrm{eV}$ can be attributted to some minor improvements that still need to be included in the model. The electronic Coriolis would couple the ${ }^{3} \Pi_{y}$ to the $2 \times 2$ manifold, yielding broader and less intense peaks. The Renner-Teller coupling for the $\mathrm{OHF}^{-}\left({ }^{2} \Pi\right)$ should also vary the width and position of some of the peaks.

\section{CONCLUSIONS}

In this work coupled diabatic potential energy surfaces are obtained for describing the $\mathrm{OH}\left({ }^{2} \Pi\right)+\mathrm{F}\left({ }^{2} P\right) \rightarrow \mathrm{O}\left({ }^{3} P\right)$ $+\operatorname{HF}\left({ }^{1} \Sigma^{+}\right)$reaction. An energy-based diabatization method is presented based on a simple electronic model for this reaction, which neglects couplings among states whose helicities differ in more than 1 . The nonadiabatic couplings close to the conical intersections are calculated numerically using the MOLPRO package ${ }^{44}$ and transformed to the diabatic representation. It is found that the present diabatized states are regularized, i.e., they do not present the singularity in the residual derivative couplings. 
The model is entirely based on three $a b$ initio eigenvalues, $1^{3} A^{\prime \prime}, 2{ }^{3} A^{\prime \prime}$, and $1^{3} A^{\prime}$, and does not need any adjustable parameter. A collection of $\approx 4000$ points has been calculated and an interpolation was performed to obtain a set of coupled diabatic PESs in all the configuration space. Such procedure gives quite accurate description of the adiabatic PESs at conical intersections and along the reaction path, yielding a significant improvement with respect to previous adiabatic fits. ${ }^{24,25}$

Angular resolved photodetachment cross sections are simulated using a wave packet method in the adiabatic and diabatic representations. For electron kinetic energies larger than $0.8 \mathrm{eV}$, all the structures appearing in the experimental spectra at $213 \mathrm{~nm}$ (Refs. 31 and 32) are reproduced. It is found that the detached electrons should present $\lambda=0$ and 1 helicities to obtain such good agreement. The small differences are attributed to electronic Coriolis couplings among the neutral OHF electronic states and Renner-Teller couplings in the $\mathrm{OHF}^{-}\left({ }^{2} \Pi\right)$ anion, both neglected in the present study.

For electron kinetic energies below $0.7 \mathrm{eV}$, the agreement is very poor because the contribution from the singlet states [correlating to the $\mathrm{O}\left({ }^{1} D\right)+\mathrm{HF}\left({ }^{1} \Sigma^{+}\right)$and $\mathrm{O}\left({ }^{3} P\right)$ $+\mathrm{HF}\left({ }^{3} \Pi\right)$ asymptotes] is very important, as shown previously using two-dimensional surfaces for the first six singlet states. $^{26}$ Three-dimensional study for the first five singlet states, correlating to the $\mathrm{O}\left({ }^{1} D\right)+\operatorname{HF}\left({ }^{1} \Sigma^{+}\right)$asymptote, is now in progress.

There are significant differences in the individual spectra obtained for each final electronic state. However, after combining them, the total angular resolved spectra obtained in the diabatic and adiabatic representations are of similar quality, showing that these quantities are not very sensitive to the nonadiabatic effects arising near conical intersections. Reaction dynamics should be affected by nonadiabatic effects since the wave packet should cross the conical intersection seams. Thus, particularly interesting are the recent experimental studies of Deyerl and Continetti ${ }^{33}$ and Continetti ${ }^{34}$ in which electron and neutral fragments are detected in coincidence. Final distribution of fragments should show larger nonadiabatic effects, specially if angular resolved quantities are obtained. Moreover, detecting $\mathrm{O}$ atoms would allow the separation of the contributions from singlets and triplets even when the $\mathrm{OH}\left({ }^{2} \Pi\right)+\mathrm{F}\left({ }^{2} P\right)$ asymptote is open. A detailed simulation of final distribution of fragments is now in progress.

Also, it is interesting to analyze the role of nonadiabatic effects in reactive collisions. In particular, recent classical and quantum calculations performed using three-dimensional adiabatic PESs (Ref. 25) yielded too low rate constants as compared with the available experimental ones. ${ }^{27}$ The inclusion of nonadiabatic effects, through the use of the diabatic representation developed in this work, could explain such disagreement. Moreover, the adiabatic PESs obtained in this work show a lower reaction barrier than in the previous work, ${ }^{24,25}$ which could produce an increase in the reaction rate constants.

\section{ACKNOWLEDGMENTS}

We want to acknowledge Professor D. M. Neumark and Professor R. E. Continetti for interesting discussions and for providing us with results prior to publication. This work has been supported by DGICYT (Ministerio de Educación y Ciencia, Spain) under Grant Nos. CTQ2004-02415/BQU and CTQ2004-06615/BQU.

${ }^{1}$ C. Jouvet, M. Boivineau, M. C. Duval, and B. Soep, J. Phys. Chem. 91, 5416 (1987)

${ }^{2}$ M. Dantus, R. M. Bowman, M. Gruebele, and A. H. Zewail, J. Chem. Phys. 91, 7437 (1989).

${ }^{3}$ N. F. Scherer, C. Sipes, R. B. Bernstein, and A. H. Zewail, J. Chem. Phys. 92, 5239 (1990).

${ }^{4}$ S. K. Shin, Y. Chen, S. Nckolaisen, S. W. Sharpe, R. A. Beaudet, and C. Wittig, Adv. Photochem. 16, 249 (1991)

${ }^{5}$ R. B. Metz, S. E. Bradforth, and D. M. Neumark, Adv. Chem. Phys. 81, 1 (1992).

${ }^{6}$ D. M. Neumark, Annu. Rev. Phys. Chem. 43, 153 (1992).

${ }^{7}$ K. Liu, J. C. Polanyi, and S. Yang, J. Chem. Phys. 98, 5431 (1993).

${ }^{8}$ J.-M. Mestdagh, B. Soep, M.-A. Gaveu, and J.-P. Visitcot, Int. Rev. Phys. Chem. 22, 285 (2003).

${ }^{9}$ L. D. A. Siebbeles, M. Glass-Maujean, O. S. Vasyutinskii, J. A. Beswick, and O. Roncero, J. Chem. Phys. 100, 3610 (1994).

${ }^{10}$ R. P. Wayne, Chemistry of Atmospheres, 2nd ed. (Clarendon, Oxford, 2000).

${ }^{11}$ A. R. Ravishankara, G. Smith, R. T. Watson, and D. D. Davis, J. Phys. Chem. 81, 2220 (1977)

${ }^{12}$ R. G. Macdonald and C. B. Moore, J. Chem. Phys. 68, 513 (1978).

${ }^{13}$ D. J. Rakestraw, K. G. McKendrick, and R. N. Zare, J. Chem. Phys. 87, 7341 (1987).

${ }^{14}$ K. Mahmud, J.-S. Kim, and A. Fontijn, J. Phys. Chem. 94, 2994 (1990).

${ }^{15}$ R. Zhang, W. J. van der Zande, M. J. Bronikowski, and R. N. Zare, J. Chem. Phys. 94, 2704 (1991).

${ }^{16}$ H. Koizumi, G. C. Schatz, and M. S. Gordon, J. Chem. Phys. 95, 6421 (1991).

${ }^{17}$ B. Ramachandran, E. A. Schrader III, J. Senekowitsch, and R. E. Wyatt, J. Chem. Phys. 111, 3862 (1999).

${ }^{18}$ F. J. Aoiz, L. Bañares, J. F. Castillo, M. Menéndez, and J. E. Verdasco, Phys. Chem. Chem. Phys. 1, 1149 (1999).

${ }^{19}$ K. Nobusada, H. Nakamura, Y. Lin, and B. Ramachandran, J. Chem. Phys. 113, 1018 (2000).

${ }^{20}$ T. Xie, J. M. Bowman, K. A. Peterson, and B. Ramachandran, J. Chem. Phys. 119, 9601 (2003).

${ }^{21}$ B. Ramachandran and K. A. Peterson, J. Chem. Phys. 119, 9590 (2003).

${ }^{22}$ S. E. Bradforth, D. W. Arnold, R. B. Metz, A. Weaver, and D. M. Neumark, J. Phys. Chem. 95, 8066 (1991).

${ }^{23}$ R. N. Dixon and H. Tachikawa, Mol. Phys. 97, 195 (1999).

${ }^{24}$ S. Gómez-Carrasco, L. González-Sánchez, A. Aguado, O. Roncero, J. M. Alvariño, M. L. Hernández, and M. Paniagua, J. Chem. Phys. 121, 4605 (2004).

${ }^{25}$ S. Gómez-Carrasco, O. Roncero, L. González-Sánchez, M. L. Hernández, J. M. Alvariño, M. Paniagua, and A. Aguado, J. Chem. Phys. 123, 114310 (2005).

${ }^{26}$ L. González-Sánchez, S. Gómez-Carrasco, A. Aguado, M. Paniagua, M. L. Hernández, J. M. Alvariño, and O. Roncero, J. Chem. Phys. 121, 9865 (2004).

${ }^{27}$ C. D. Walter and H. G. Wagner, Ber. Bunsenges. Phys. Chem. 87, 403 (1983).

${ }^{28}$ A. Weaver, R. B. Metz, S. E. Bradforth, and D. M. Neumark, J. Chem. Phys. 90, 2070 (1989).

${ }^{29}$ M. Mayer, L. S. Cederbaum, and H. Köppel, J. Chem. Phys. 100, 899 (1994).

${ }^{30}$ S. Mahapatra, H. Köppel, and L. S. Cederbaum, J. Chem. Phys. 110, 5691 (1999)

${ }^{31}$ D. M. Neumark, Phys. Chem. Chem. Phys. 7, 433 (2005).

${ }^{32}$ S. E. Bradforth, Ph.D. Thesis, University of California, Berkeley, 1992.

${ }^{33}$ H.-J. Deyerl and R. E. Continetti, Phys. Chem. Chem. Phys. 7, 855 (2005).

${ }^{34}$ R. E. Continetti (private communication).

${ }^{35}$ H. Köppel, in Conical Intersections: Electronic Structure, Dynamics and Spectroscopy, Advanced Series in Physical Chemistry, edited by D. R. 
Yarkony, W. Domcke, and H. Köppel (World Scientific, Singapore, 2004), p. 175.

${ }^{36}$ F. T. Smith, Phys. Rev. 179, 111 (1969).

${ }^{37}$ M. Baer, Chem. Phys. Lett. 35, 112 (1975).

${ }^{38}$ M. Baer, Mol. Phys. 40, 1011 (1980).

${ }^{39}$ C. A. Mead and D. G. Truhlar, J. Chem. Phys. 77, 6090 (1982).

${ }^{40}$ A. Thiel and H. Köppel, J. Chem. Phys. 110, 9371 (1999).

${ }^{41}$ M. Baer, Chem. Phys. Lett. 347, 149 (2001).

${ }^{42}$ H. J. Werner and W. Meyer, J. Chem. Phys. 74, 5802 (1981).

${ }^{43}$ D. Simah, B. Hartke, and H.-J. Werner, J. Chem. Phys. 111, 4523 (1999).

${ }^{44}$ H.-J. Werner, P. J. Knowles, J. Almlöf et al., MOLPRO, a package of $a b$ initio programs, Version 2002.7.

${ }^{45}$ H.-J. Werner and P. J. Knowles, J. Chem. Phys. 89, 5803 (1988).

${ }^{46}$ H.-J. Werner and P. J. Knowles, Chem. Phys. Lett. 145, 514 (1988).

${ }^{47}$ D. A. Varshalovich, A. N. Moskalev, and V. K. Khersonskii, Quantum Theory of Angular Momentum (World Scientific, Singapore, 1988).

${ }^{48}$ H. Köppel, W. Domcke, and L. S. Cederbaum, Adv. Chem. Phys. 57, 59 (1984).

${ }^{49}$ T. H. Dunning, Jr., J. Chem. Phys. 90, 1007 (1989).

${ }^{50}$ R. A. Kendall, T. H. Dunning, Jr., and R. J. Harrison, J. Chem. Phys. 96, 6769 (1992).
${ }^{51}$ H.-J. Werner and P. J. Knowles, J. Chem. Phys. 82, 5053 (1985).

${ }^{52}$ H.-J. Werner and P. J. Knowles, Chem. Phys. Lett. 115, 259 (1985).

${ }^{53}$ E. R. Davidson, J. Comput. Phys. 17, 87 (1975).

${ }^{54}$ A. Aguado and M. Paniagua, J. Chem. Phys. 96, 1265 (1992).

${ }^{55}$ M. Abramowitz and I. A. Stegun, Handbook of Mathematical Functions (Dover, New York, 1972).

${ }^{56}$ W. H. Press, S. A. Teukolsky, W. T. Vetterling, and B. P. Flannery, Numerical Recipes (Cambridge University Press, Cambridge, 1994).

${ }^{57}$ J. C. Tully, R. S. Berry, and B. J. Dalton, Phys. Rev. 176, 95 (1968).

${ }^{58}$ J. Cooper and R. N. Zare, J. Chem. Phys. 48, 942 (1968).

${ }^{59}$ J. L. Dehmer and D. Dill, Phys. Rev. A 18, 164 (1978).

${ }^{60}$ F. Rebentrost and W. A. Lester, Jr., J. Chem. Phys. 64, 3879 (1976).

${ }^{61}$ C. Jouvet and J. A. Beswick, J. Chem. Phys. 86, 5500 (1987).

${ }^{62}$ M.-L. Dubernet and J. Hutson, J. Chem. Phys. 101, 1939 (1994).

${ }^{63}$ M. H. Alexander, D. E. Manolopoulos, and H.-J. Werner, J. Chem. Phys. 113, 11084 (2000).

${ }^{64}$ L. González-Sánchez, S. Gómez-Carrasco, A. Aguado, M. Paniagua, M. L. Hernández, J. M. Alvariño, and O. Roncero, J. Chem. Phys. 121, 309 (2004).

${ }^{65}$ N. Elghobashi and L. González, J. Chem. Phys. 124, 174308 (2006). 